\title{
Ultrasmooth thin film and nano-scale structure fabrication to extend the bounds of optical microscopy
}

by

Eden Rafealov

\author{
A thesis \\ submitted to the Victoria University of Wellington \\ in fulfilment of the \\ requirements for the degree of \\ Master of Engineering \\ in Electronic and Computer System Engineering. \\ Victoria University of Wellington \\ 2016
}



For mum and $a b a$. 



\begin{abstract}
Plasmonic devices including superlenses, hyperlenses, and far-field superlenses are specially fabricated elements which can improve the resolution of optical microscopy past inherent theoretical limits. However, their fabrication is extremely difficult as they often require ultrasmooth thin films and finely structured silver (Ag). While Ag has an ideal response for these types of lenses, its fabrication in such devices is challenging. Hence, this thesis investigates viable methods of producing ultrasmooth Ag thin films and nano-scale Ag features in order to advance research in plasmonic devices.

Optimum plasmon response requires the fabrication of ultrasmooth thin silver films, which presents several challenges, such as high surface roughness and high optical loss. Thin $1 \mathrm{~nm}$ seed layers were fabricated in advance of Ag layers in order to improve the surface properties of $\mathrm{Ag}$. We found that a $1 \mathrm{~nm}$ germanium (Ge) seed layer results in a $400 \%$ reduction in surface roughness down to $0.64 \mathrm{~nm}_{\mathrm{RMS}}$, but offers increased optical loss by about 3\% over Ag alone. However, an inert atmosphere high temperature anneal of a $\mathrm{Ge} / \mathrm{Ag}$ stack results in preferential grain growth, further reducing surface roughness to $0.61 \mathrm{~nm}_{\mathrm{RMS}}$, while also improving transmission by up to $14 \%$ over Ag alone. Similar procedures were conducted on copper $(\mathrm{Cu})$ and silver oxide $\left(\mathrm{AgO}_{\mathrm{x}}\right)$ seed layers. While $\mathrm{Cu}$ results in very smooth Ag films of $0.61 \mathrm{~nm}_{\mathrm{RMS}}$ for films $<10 \mathrm{~nm}$ thick, performance deteriorates at $\mathrm{Ag}$ thicknesses above $10 \mathrm{~nm}$, which are preferred for the plasmonic applications identified above. Furthermore, $\mathrm{AgO}_{\mathrm{x}}$ produces very rough surfaces on substrates which are amorphous-a property which is essential for our use. However, $\mathrm{AgO}_{\mathrm{x}}$ on crystalline substrates produced
\end{abstract}


smooth surfaces of $0.3 \mathrm{~nm}_{\mathrm{RMS}}$ and may be useful for other plasmonic applications.

Interference lithography (IL) was selected as the method to create the periodic nano-scale structures. The IL equipment was modified with the addition of bandpass light filters, $5 \mu \mathrm{m}$ pinhole Fourier filters, and air vortex shields. Also, elimination of both external vibration and time-dependant vacuum lines are included. With this IL environment, we were able to produce periodic gratings anywhere from $1 \mu \mathrm{m}-300 \mathrm{~nm}$ pitch through rigorous optimisation of photoresist, exposure, and development processes. Ultimately, this lead to the fabrication of high contrast, $200 \mathrm{~nm}$ period gratings for use in a far-field superlens. The designs and procedures outline within will result in increased performance and production of far-field superlenses with limited equipment, therefore facilitating increased performance of optical microscopes. 


\section{Acknowledgments}

I would like to give my special thanks to my advisors, Ciaran Moore and Gideon Gouws, for their support in my research. I greatly appreciate the guidance, patience, and encouragement to help me complete this thesis.

To my office-mates, I couldn't have survived many late nights of experimentation and writing without your friendship and offerings of food.

To my friends who made the seldom weekend off something great, and a necessary break from study.

Finally, to my family, who's support throughout has been unwavering and unfathomably cherished, without which this would have not been possible, thank you.

For those who remain unnamed, I apologise and am truly grateful. 


\section{Contents}

1 Introduction 1

2 Background $\quad 5$

2.1 Optical Microscopes ................ 5

2.1 .1 Optical resolution . . . . . . . . . . . . 6

2.2 Evanescent modes . . . . . . . . . . . . . . . 9

2.2.1 Negative Index Materials . . . . . . . . . . . . . . . 9

2.3 Surface plasmon polariton . . . . . . . . . . . . . 11

2.3.1 Surface plasmons on rough/corrugated surfaces . . . 13

2.4 Silver thin film . . . . . . . . . . . . . . . . . 14

2.5 Nano-scale diffraction grating . . . . . . . . . . . . . . . . . . . . . . . . . .

2.6 Fabrication issues . . . . . . . . . . . . . . 16

2.6.1 Surface roughness . . . . . . . . . . . 17

2.6.2 Grating size, shape, and roughness . . . . . . . . . 18

2.7 Literature Survey . . . . . . . . . . . . . . . . . 20

2.7.1 Surface roughness . . . . . . . . . . . 20

2.7.2 Nano-scale diffraction grating fabrication . . . . . . . 21

3 Photonic structure design 23

3.1 Silver thin film properties . . . . . . . . . . . . . 23

3.1 .1 Seed layers . . . . . . . . . . . . . . . . 24

3.1 .2 Silver oxide seed layer . . . . . . . . . . . . 25

3.2 Grating fabrication process ............. 26 
3.2.1 Photoresist. . . . . . . . . . . . 26

3.2.2 Mask aligner . . . . . . . . . . . . 26

3.2.3 Interference lithography . . . . . . . . . . . . 28

3.2.4 Development . . . . . . . . . . . . 31

3.2.5 Image reversal photoresist . . . . . . . . . . . 32

3.2.6 Grating quality and optimisation . . . . . . . . . . 34

3.3 Far field superlens design . . . . . . . . . . . . . 35

4 Plasmonic film fabrication $\quad 39$

4.1 Thin film fabrication . . . . . . . . . . . . . . 39

4.2 Germanium seed layer . . . . . . . . . . . . . . . . 40

4.3 Copper seed layer . . . . . . . . . . . . . . . . . 43

4.4 Silver oxide seed layer . . . . . . . . . . . . . . . 46

5 Grating design $\quad 49$

5.1 Metalisation and lift-off . . . . . . . . . . . . . 52

5.2 Nano-scale grating idiosyncrasies . . . . . . . . . . 55

6 Conclusions and future work $\quad 61$

6.1 Future work . . . . . . . . . . . . . . 62

6.1.1 Film efficiency and evanescent mode production . . . 62

6.1.2 Nano-scale diffraction quality . . . . . . . . . . . . 64

6.1 .3 Far field superlens ... . . . . . . . . 65 


\section{Nomenclature}

$\begin{array}{ll}n & \text { refractive index } \\ x, y, z & \text { cartesian coordinates } \\ s & \text { photon momentum change } \\ \lambda & \text { wavelength of light } \\ \theta & \text { angle of light } \\ \hbar & \text { reduced Plank constant } \\ \varepsilon & \text { electric permittivity } \\ \mu & \text { magnetic permeability } \\ E & \text { electric field } \\ k & \text { free space wavevector } \\ \omega & \text { frequency } \\ t & \text { time } \\ H & \text { magnetic field } \\ \beta & \text { surface plasmon propagation constant } \\ \Lambda & \text { grating wavenumber } \\ p & \text { grating period } \\ m & \text { diffraction order } \\ S_{a} & \text { average surface roughness } \\ S_{q} & \text { RMS surface roughness } \\ \rho & \text { resistivity } \\ d & \text { dose } \\ I_{\text {mirror }} & \text { power density at mirror } \\ I_{\text {pattern }} & \text { power density at sample } \\ I_{\text {sample }} & \text { power density incident to sample } \\ P_{\text {sample }} & \text { instantaneous power to the sample } \\ A & \text { area } \\ R_{\text {mirror }} & \text { reflectivity of Lloyd's mirror } \\ & \end{array}$


FSL far field superlens

EBL electron-beam lithography

FIB focused ion beam milling

IL interference lithography

NIM negative index material

PIM positive index material

SPR surface plasmon resonance

EM electromagnetic

SP surface plasmon

SPP surface plasmon polariton

Ag silver

EME evanescent mode enhancement

NSDG nano-scale diffraction grating

RMS root-mean squared

AFM atomic force microscope

LWR line width roughness

LER line edge roughness

$\mathrm{Ti}$ titanium

$\mathrm{Ni}$ nickel

Ge germanium

$\mathrm{Cu} \quad$ copper

$\mathrm{AgO}_{x}$ silver oxide

$\mathrm{SiO}_{2} \quad$ silicon oxide

$\mathrm{O}_{2} \quad$ oxygen

UV ultraviolet

$\mathrm{He}-\mathrm{Cd}$ helium-cadmium

$\mathrm{DiH}_{2} \mathrm{O}$ de-ionised water

SEM scanning electron microscope

IRP image reversal photoresist

PEB post-exposure bake 
RRP resist reflow process

PMMA polymethyl methacrylate

IPA isopropyl alcohol

RF radio frequency

SCCM standard cubic centimetres per minute

$\mathrm{N}_{2} \quad$ (pressurised) nitrogen

PGMEA propylene glycol monomethyl ether acetate

NMP n-methyl-2-pyrrolidone

BARC bottom anti-reflective coating

FFT fast-fourier transform

$\mathrm{He}^{-} \mathrm{O}_{2}$ helium-oxygen

RIE reactive ion etch

PVA polyvinyl alcohol

DAPI 4',6-diamidino-2-phenylindole

Cr chromium

$\mathrm{SF}_{6} \quad$ sulfur hexafluoride 


\section{List of Figures}

2.1 Airy pattern which can result from optical microscopes. . . . 7

2.2 Resolution comparison of Abbe and Rayleigh criterion. . . . 8

2.3 Comparison of propagating waves and evanescent modes . 9

2.4 Comparison of material indicies and their optical effects. . . 10

2.5 Concept of surface plasmons polariton with wave vector. . . 11

2.6 Dispersion relation of bulk and surface plasmons which are separated by the light-line and band-gaps imposed by $\omega$ constraints. . . . . . . . . . . . . . . 13

2.7 Evanescent mode decay, and enhancement through superlens. 15

2.8 Concept of near-field to far-field transition. . . . . . . . . . 16

2.9 Concept of stylus operated profilometers, illustrating measurement of surface roughness. . . . . . . . . . . . 18

2.10 Illustration of LWR, and development of polymer aggregate. 19

3.1 Simulated resist profile from mask aligner. . . . . . . . . . . 27

3.2 Interference lithography system setup . . . . . . . . . . . . 28

3.3 Illustration of terms used in IL dosage calculations derived from impinging light on Lloyd's mirror stage. . . . . . . . . . 29

3.4 Comparison of over- and under-developed photoresist profiles. . . . . . . . . . . . . . . 31

3.5 Simplified image reversal fabrication steps. . . . . . . . . 33

3.6 Comparison of positive photoresist and IRP profile after metalisation. . . . . . . . . . . . . . . 34 
3.7 Comparison of over- and under-developed IRP photoresist profiles. . . . . . . . . . . . . . 34

3.8 Increased RRP illustrates rounding of gratings. . . . . . . . . 35

3.9 Comparison of similar FSL designs. . . . . . . . . . . 36

3.10 Simplified fabrication steps for the FSL. . . . . . . . . . 37

4.1 Comparison of AFM profile scans of $\mathrm{Ag}$ film, and $\mathrm{Ge} / \mathrm{Ag}$ film on borosilicate. . . . . . . . . . . . . . . . 41

4.2 Ag surface roughness and transmission with Ge seed layer. . 42

4.3 Roughness of $\mathrm{Ge} / \mathrm{Ag}$ films with increasing annealing temperature for 10 and $40 \mathrm{~nm}$ thick Ag films. . . . . . . . . . . 43

4.4 Ag surface roughness and SEM surface and profile views of Ge and Cu. . . . . . . . . . . . . . . . . . . . . 44

4.5 Surface roughness of $\mathrm{Cu} / \mathrm{Ag}$ films and \% transmission with annealing temperature. . . . . . . . . . . . . 45

4.6 SEM comparison of $\mathrm{Ag}$ with no seed, $\mathrm{AgO}_{\mathrm{x}}$ seed, and $\mathrm{Ge}$ seed. . . . . . . . . . . . . . . . 47

4.7 AFM micrographs of $\mathrm{AgO}_{\mathrm{x}}$ deposited on borosilicate and sapphire. . . . . . . . . . . . . . . . . 4 48

5.1 Spin speed curves for AZ 1500 resist products. . . . . . . . . 50

5.2 SEM images of dilute AZ 1518 gratings. . . . . . . . . . . . . 51

5.3 SEM images showing $1 \mu \mathrm{m}$ gratings fabricated using AZ 1518, AZ 5214E, and AZ 701MiR. . . . . . . . . . . 53

5.4 Illustration and fabrication results of nonideal lift-off . . . . 54

5.5 Comparison of initial lift-off technique, and optimised liftoff technique resulting. . . . . . . . . . . . . . 55

5.6 Exposure and development of $300 \mathrm{~nm}$ pitch NSDGs with and without BARC. . . . . . . . . . . . . 57

5.7 Photoresist gratings with and without vacuum and vortex exposure issues. . . . . . . . . . . . . . 58

5.8 BARC etching with $\mathrm{He}_{-} \mathrm{O}_{2}$ plasma on $1 \mu \mathrm{m}$ and $200 \mathrm{~nm}$ pitch. 59 
6.1 Simplified illustration of Kretchmann configuration with evanescent dye. . . . . . . . . . . . . . . . . 63 63

6.2 Spectrometer response from fluoro-Kretchmann setup. . . . 64

6.3 Simplified fabrication steps for the FSL. . . . . . . . . . . 66 


\section{Chapter 1}

\section{Introduction}

Optical microscopes are essential scientific tools to observe objects which are too small to be seen with the naked eye. They provide a simple and accessible way for researchers to study the existence of cells, make medical diagnoses, and fabricate electronic circuits. However, traditional optical microscopes are limited by a fundamental minimum resolution, known as the diffraction limit. As a result, they cannot observe features smaller than approximately half the wavelength of light. For visible light, the best performing optical microscopes reach this limit when trying to distinguish features roughly $300 \mathrm{~nm}$ apart [1].

Plasmonics is a fairly new area of interest and covers the extraordinary properties of metals, specifically their electromagnetic field and free electron interactions [2]. The free electrons can interact with the electric component of incident electromagnetic waves producing plasmon oscillation [3]. These plasmonic optical traits can be used to produce a superlens [4] made from planar slabs of materials with refractive indicies of $n=-1$ and are used to overcome the diffraction limit. Superlenses focus all incident spatial frequency components of a point source, and enhancing sub-wavelength feature information in order to attain a higher resolution image, effectively beating the diffraction limit.

While superlens properties are useful through thin metal films, the practicality of producing these films with low surface roughness pose is- 
sues in its viability as a plasmonic device. Surface roughness interacts negatively with plasmonic resonance, resulting in losses; however, surface roughness itself is difficult to reduce in thin metal films. Attempts have been made to smooth metal films typically using seed layers $[5,6]$ to promote a more ideal layer-by-layer growth of the metal, resulting in lower surface roughness. However, they have yet to be correctly implemented with superlens design [7]. Therefore, in order to viably create a superlens, focus must be placed on producing smooth films. Smooth superlens configurations can allow for sub-diffraction image reconstruction, but the position where the reconstruction takes place is not easily observed.

A far-field superlens (FSL) [8] builds on superlens design with the addition of nano-scale diffraction gratings. These gratings add frequency shifting through the inherent Moire fringes that occur where the image from the superlens, and nano-scale diffraction gratings meet. This allows for a sub-wavelength image to be realised far away (on the order of microns) from the FSL using an optical microscope. FSL performance is limited mainly by surface roughness, line edge roughness, grating pitch, and film thickness. By improving any one, or more of these components, the FSL performance will inherently improve. These FSL qualities are vital in its repeatability and accuracy as a lens.

Fabrication of FSL components tend to require very specialised equipment such as electron-beam lithography (EBL) or focused ion beam milling (FIB) because of the sub-wavelength fabrication preconditions. These techniques impose inherent restrictions on cost, ease of fabrication, and time. Interference lithography (IL), while less common, has the potential to match EBL/FIB quality while forgoing their shortcomings.

As a result, this thesis covers the inherent FSL issues, the research aims to greatly reduce surface roughness of FSL components, and increase uniformity to improve imaging. Furthermore, optimisation of fabrication techniques and exposure environments of IL systems are developed in order to viably create diffraction gratings at $<200 \mathrm{~nm}$ pitch. The benefits 
of the design and fabrication methods proposed herein include new and easier optical studies of natural and engineered nano-scale objects that are not possible using traditional optical microscopes. This project has the potential to affect applications such as electronics manufacturing, biomedical sensing and optical imaging.

The structure of this thesis is as follows: Chapter 2 covers a background in photonic optics and FSL theory. In addition, the challenges of fabrication are also mentioned, and a review of photonic devices such as superlens and FSL research is summarised. Chapter 3 covers strategies in overcoming fabrication issues while also introducing additional steps to aid in the increasing performance of FSLs. The implementation of these designs is detailed in Chapters 4 and 5. Finally, Chapter 6 provides a summary of our findings and future work recommendations are also presented. 


\section{Chapter 2}

\section{Background}

This chapter introduces the concepts that govern optical microscopy and discusses some of the inherent issues with typical microscopy techniques. Details of the components and phenomena which make sub-wavelength imaging possible are then provided. Finally, literature regarding these established techniques and technology is analysed.

\subsection{Optical Microscopes}

Optical microscopes magnify and focus objects through the use of glass lenses in order to produce a well lit image. These microscopes can typically provide very high magnification; however, because of the inherent limitations of light, they can only resolve object detail to approximately $300 \mathrm{~nm}$. The diffraction limit, comes from the wave nature of light and can be described by two closely related values: the Abbe and Rayleigh criteria [9]. The Abbe criterion is derived from Heisenberg's uncertainty principle [10]:

$$
\Delta x \Delta s \geqslant \frac{1}{2} \hbar,
$$

where $\Delta s$ is photon momentum change, $s=(\hbar n / \lambda) \sin \theta, \lambda$ is the work- 
ing wavelength of light, $n$ is the refractive index of the medium the light travels though, $\theta$ the acceptance angle of the detection system, $\Delta x$ is the precision of location, or resolvable difference in distance, and $\hbar$ the reduced Planck constant. Hiesenberg's uncertainty principle describes the fundamental limit of precision with which particle properties are known, such as position. A simplification and reduction of Equation 2.1 leads to the Abbe criterion:

$$
\Delta x \geqslant \frac{\lambda}{2 n \sin \theta} .
$$

The Rayleigh criterion is closely defined [11] :

$$
\Delta x \geqslant 0.61 \frac{\lambda}{n \sin \theta}
$$

These criteria mean that given $\lambda=500 \mathrm{~nm}$ (visible light), $n=1$ (air), and $\theta=90^{\circ}$ (i.e., light enters normal to the detection system), then resolvable distance, $\Delta x$, is limited to a minimum of $305 \mathrm{~nm}$.

Light waves are diffracted and scattered as they travel through a microscope. This causes a loss of information regarding the exact location of a point source, which can be light from a specific feature of an object. This loss of information results in an intensity distribution, known as an Airy pattern $^{1}$ [13]. As illustrated in Figure 2.1, these Airy patterns produce a diffuse, delocalised pattern which causes interference with nearby object intensities and leads to the difficulty of resolving separate objects, and the inherent resolution limit of optical microscopes.

\subsubsection{Optical resolution}

The discrepancy between the Abbe and Rayleigh criteria stems from separate definitions for resolving two objects from one another, and the loca-

\footnotetext{
${ }^{1}$ Airy patterns are well modelled by Bessel functions. The Rayleigh criterion accounts for these Airy patterns through the 0.61 coefficient term which approximates the first zero of the Bessel function of the first kind [12].
} 


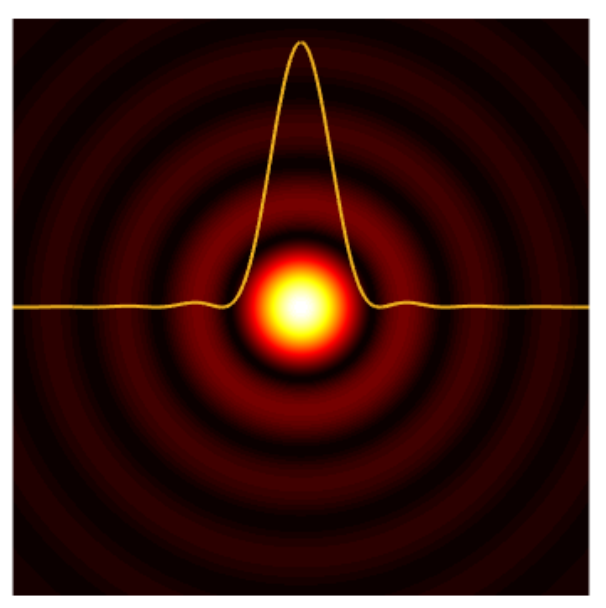

Figure 2.1: Top-down example of Airy pattern which can result from image detection through an optical microscope. Orange overlay shows the profile of the Airy function.

tion with which the image is resolved. Optical resolution can be thought of as the minimum separation between two point-sources in order to distinguish the original sources upon reconstruction of an image; however, this interpretation still allows for two definitions to arise, where objects can be distinguishable, and barely distinguishable, where the latter definition comes from a smaller, but sufficient distance between two objects. These definitions arise from the inherent difficulty to define the border at which a resolved image is produced from one object, or multiple objects which are indistinguishable from one another. The Rayleigh and Abbe criteria resolution definitions are illustrated in Figure 2.2.

It is possible to see the two objects in Figure 2.2(c), whose separation is below the diffraction limit, produce a single maximum from their reconstruction. This means their image becomes indistinguishable upon reconstruction. The objects in Figures 2.2(a) and (b), which are further apart, produce noticeable peaks in their reconstruction and hence can be separately distinguished as more than one source object, as the distance between them is larger than the diffraction limit. 


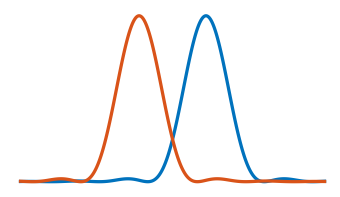

(a)

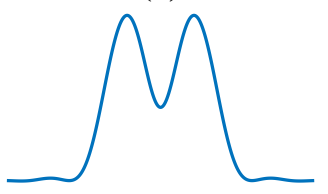

(d)

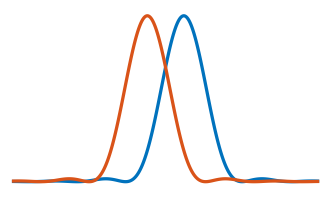

(b)

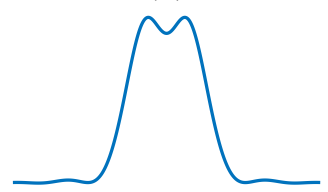

(e)

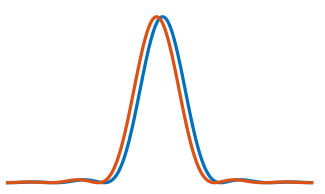

(c)

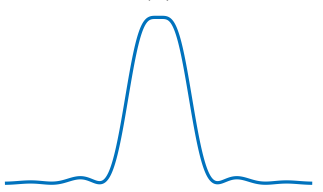

(f)

Figure 2.2: Various illustrations of resolution limits. The Rayleigh criterion (d) is described by the reconstruction of the sum of two close objects in (a), producing two distinct peaks. The Abbe criterion (e) is described by the sum of two overly close objects in (b), producing two small, but discernible peaks. Finally, (f) shows the sum of almost overlapping objects (c), producing one single peak upon reconstruction.

The Rayleigh criterion is most commonly used to express the diffraction limit in the optical far-field, whereas the Abbe criterion does so in the near-field [14]. As this thesis covers superlens fabrication for applications in the far-field, the Rayleigh criterion will be adopted as the predominant standard resolvable distance between two objects.

The diffraction limit imposed by the Rayleigh criteria holds true only when pure propagating waves radiated by an object are used to reconstruct its image. Light from an object's large features is carried through these propagating waves, whereas the very small feature components are carried by evanescent modes, which decay at an exponential rate as they travel away from the object. The quick decay of these modes results in a loss of fine detail information at the far-field [15]. 


\subsection{Evanescent modes}

One way to beat the diffraction limit which the Rayleigh criterion imposes is by capturing evanescent modes in an image. Evanescent modes result from the inability of electric and magnetic fields to become discontinuous at the boundary between two media. Hence evanescent modes occur at the junction of materials with differing wave motion properties, such as a difference in refractive index, $n$ [16]. As illustrated in Figure 2.3, evanescent waves decay at an exponential rate, rather than propagating sinusoidally, from the interface at which they were formed. For this reason they are difficult to capture, and are often referred to as near-field waves as they cannot advance to the far-field.

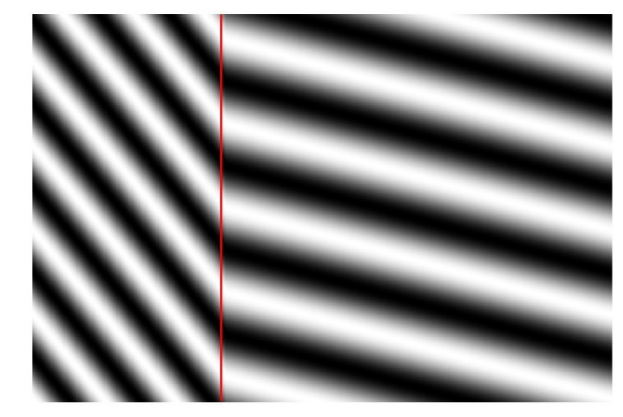

(a)

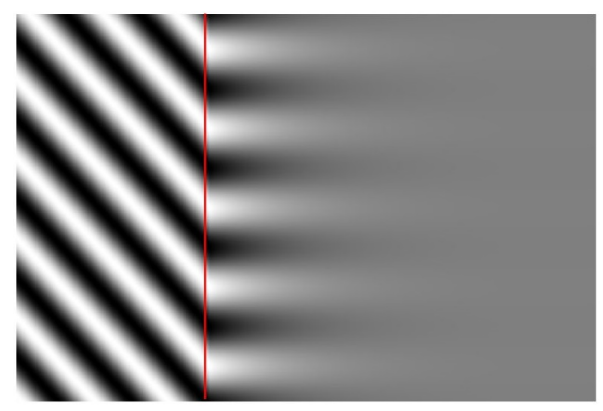

(b)

Figure 2.3: In (a) propagating waves experience a phase change at the interface (red vertical line), then continue with high contrast to the right of the image. In (b) evanescent modes contrast rapidly decay as they travel away from the interface resulting in a low contrast pattern in the right of (b). Light incident from bottom left corner [17].

\subsubsection{Negative Index Materials}

Refractive indicies of materials determine how light scatters through a material. Typical materials have a positive refractive index $(n>0)$, however 
there are some materials which exhibit negative refractive indicies at specific wavelengths. These negative index materials (NIM) display useful characteristics. Positive index material (PIM) lenses require curved surfaces for light to be focused; however, a NIM does not [18]. In fact, planar slabs of NIMs can support the focusing of evanescent modes, therefore allowing for the production of an image $[4,19]$. An example of this planar focusing can be seen in Figure 2.4(b), and is directly compared with a traditional convex lens in Figure 2.4(a).

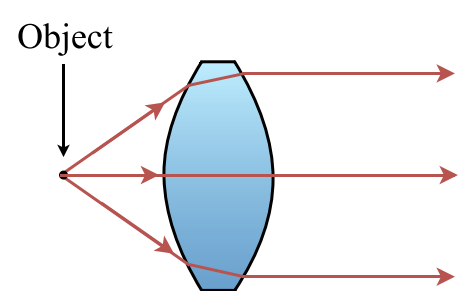

a)

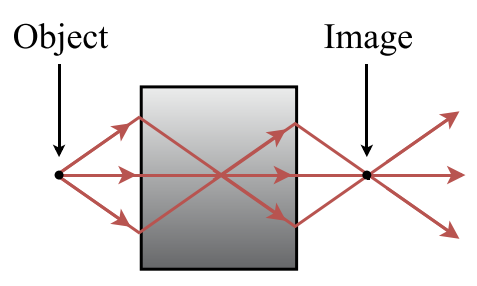

b)

Figure 2.4: Concept of traditional focusing through (a) convex lens, compared with focusing through (b) NIM.

A NIM spawns from two material properties: both electric permittivity $\varepsilon$, and magnetic permeability $\mu$ must be less than zero [19]. That being said, these two requirements impose very strict conditions on material choice and further raise the issue that magnetic activity can be difficult to detect at optical frequencies [20]. However, if the dimensions of the system are restricted to sizes smaller than the wavelength of light used as the excitation source, the magnetic and electric fields may be considered independently of one another, i.e. they are decoupled [21]. As a result, we can focus upon only the electric fields, and the magnetic component, $\mu$ can therefore be ignored. Hence, at these size constraints, a material with only negative $\varepsilon$ can be used as a good approximation of the behaviour of a NIM. A NIM which is sandwiched between dielectrics actively supports surface plasmon resonance (SPR) as it occurs at the interface between two media with $\varepsilon$ of opposite sign, such as a metal and dielectric. 


\subsection{Surface plasmon polariton}

Polarisation can be induced in a medium from incident electromagnetic (EM) waves. This polarisation and EM wave excitation interaction, described as an acoustic wave in a sea of free electronics is called polariton $[22,23]$. Furthermore, the plasmon is a charge-density oscillation describing the movement of mobile electrons ('plasma'). The plasmon can be divided into two main types: a bulk plasmon, which arises from fluctuations of the free charge density within the metal [24], and a surface plasmon (SP), which travels, along the surface of the interface between the metal and dielectric under specific conditions. The SP is described by [25]:

$$
E= \pm E_{0} e^{+i\left(k_{x} x \pm k_{z} z-\omega t\right)}
$$

where $E_{0}$ is some electric field polarised in $y, i=\sqrt{-1}, k_{x}$ and $k_{z}$ are free space wave vectors describing the magnitude and directional wave information, $x$ and $z$ are cartesian coordinates, $\omega$ is the frequency of surface oscillations, and $t$ is the time component; however, without loss of generality, $[\omega t]=0$ due to assumed time invariance. Restrictions $E_{0}>0$ for $z \geq 0, E_{0}<0$ for $z \leq 0$, and imaginary $k_{z}$ are also imposed. Together, the plasmon and the polariton form surface plasmon polariton (SPP) which describes the charge motion of the plasma and the EM waves in the dielectric [26]. Figure 2.5 illustrates an SPP wave propagating along a metaldielectric interface $\left(\varepsilon_{1} \varepsilon_{2}\right)$ in the $x$ - and $y$-directions.

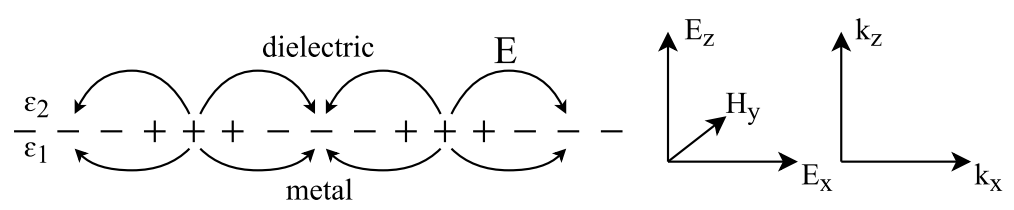

Figure 2.5: Concept of SPP propagating along a surface in $x . E_{x}$ and $E_{z}$ indicate electric fields in $x$ and $y$, respectively, and $H_{y}$ indicates magnetic field in $y$ direction. Alternating + and - indicate the the regions of higher and lower electron density, respectively. 
From Equation 2.4 it is possible to see advancement in $z$ from the surface causes the imaginary, loss term, $\left[k_{z} z\right]$ to increase, hence $E_{z}$ exponentially decays. Inherent losses in each medium, described through the imaginary loss term of permittivity, also contribute to a decay in $x$ and $y$ :

$$
\varepsilon=\varepsilon^{\prime}+i \varepsilon^{\prime \prime}
$$

A reduction in $\varepsilon^{\prime \prime}$ would result in lower loss as the plasmons traverse $x$ and $y$. This can be achieved simply by selecting materials with low imaginary permittivity, $\varepsilon^{\prime \prime}$. Additionally, these losses are wavelength dependant in nature and a specific excitation wavelength may result in lower overall losses. For example, $\varepsilon_{A g, \lambda=600 \mathrm{~nm}}^{\prime \prime}=1.08$, while $\varepsilon_{A g, \lambda=325 \mathrm{~nm}}^{\prime \prime}=0.736$ [27].

When the wave vector of the incoming EM radiation matches the wavelength of the resultant SPs, the plasma begins to 'resonate' [28]. The surface plasma propagation constant, $\beta$, at an interface such as this can be described [29]:

$$
\beta=k \sqrt{\frac{\varepsilon_{m} n_{s}^{2}}{\varepsilon_{m}+n_{s}^{2}}},
$$

where $k$ is free space wave vector of the incoming EM field, $\varepsilon_{m}$ the permittivity of the metal, and $n_{s}$ the refractive index of the dielectric media. Restrictions on $\beta>0$ are imposed, and from the term $\left[\varepsilon_{m}+n_{s}^{2}\right]$ in Equation 2.6, it is possible to see that the SPs may only be supported by an interface of media for which $\operatorname{Re}\left\{\varepsilon_{m}\right\}<-\left(n_{s}^{2}\right)$ holds true.

These many waves can be described through the dispersion relation. The dispersion relation compares visible light, plasmon, and SPP conditions, and illustrates the properties which separate each of these waves [30]. For example, the light line seen in Figure 2.6 describes the barrier between visible and attainable light on the left, and those which are not easily detected on the right. The dispersion relation of bulk and surface plasmons where the SPP curve is asymptotic in nature, reaching $\omega_{s p}$ at high $k$, can also be seen in Figure 2.6. This is in contrast to the plasmon polariton curve, where it exists at a higher $\omega$ value. As the SPP $k$ value is increased, 
the band-gap which exists between the two curves, described by $\omega_{p}-\omega_{s p}$, can be overcome. This results in the conversion of SPP to propagating SPs and photons which can be used for imaging at the far-field. That being said, the increase in $k$ must be introduced artificially such that the wavevectors of each material are matched. This artificial $k$ increase to excite propagating SPs can be produced through the use of periodic grating structures, or prism-based Kretchmann configurations, both of which are discussed later.

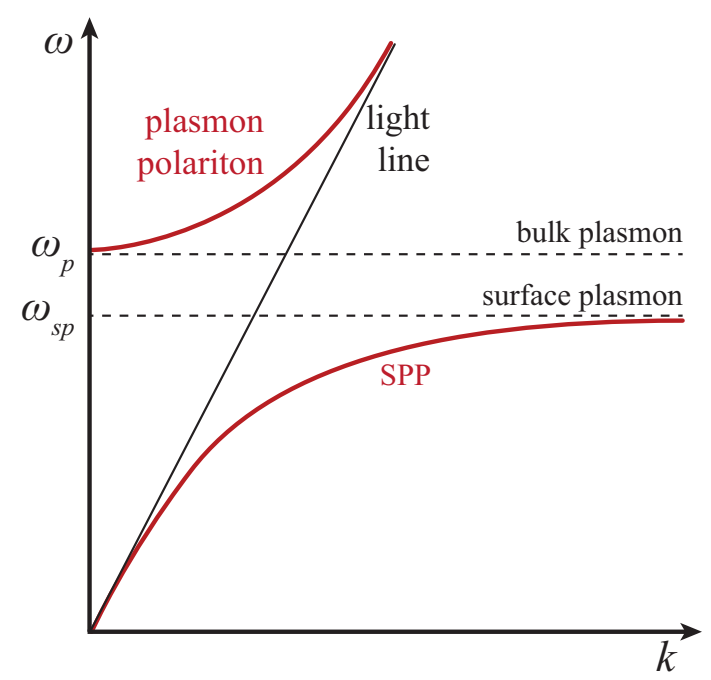

Figure 2.6: Dispersion relation of bulk and surface plasmons which are separated by the light-line and band-gaps imposed by $\omega$ constraints.

\subsubsection{Surface plasmons on rough/corrugated surfaces}

As SPs travel along a smooth surface, intensity loss and absorption of SPs is mostly dominated by the losses in Equations 2.4 and 2.5 [31]. However, if the surface is rough, an additional mechanism affects both SP losses and free space propagation distance from the surface. The travelling wave vector parallel to the surface, $k_{x}$, experiences a loss, $\Delta k_{x}$ due to the surface roughness. This seemingly unwanted loss produces a resonance transition 
of SPs into photons [32,33]. The transition compensates for the evanescent mode decay in free space, and hence enhances these modes.

This is a phenomenon which can be exploited in order to convert high spatial frequency information carried in SPs into propagating waves; however, the roughness which contributes to this conversion must be ordered and specific, such that propagating waves with equal phase are produced and interfere constructively to increase intensity.

\subsection{Silver thin film}

As $\varepsilon<0$ inherently occurs in silver (Ag) at optical wavelengths [34] an Ag thin film with ideal film thickness, surface roughness, and image plane placement effectively acts as an evanescent mode coupler. Furthermore, as $\operatorname{Re}\left\{\varepsilon_{A g}\right\}=-2.13<-1$ (assuming air as dielectric and $\lambda=325 \mathrm{~nm}$ ) Ag also supports SPR. It is studied in optics due to its novel properties, can be used to build a photonic device known as a superlens $[4,7,35,36]$, and achieves its increased performance through enhancement of evanescent modes caused by SPR. As evanescent modes carry high spatial frequency information corresponding to an object's small features, a superlens made of Ag may be employed to recover this information through evanescent mode enhancement (EME), leading to the formation of a near-field subwavelength image. This restoration is illustrated in Figure 2.7.

An issue which arises from this evanescent mode conversion is the near-field placement of the reconstructed image [37]. It should be noted that as the near-field is limited to $\lambda / 2 \pi$ [38] so is the near-field placement of the image. Given $\lambda=325 \mathrm{~nm}$, the image occurs at a maxmimum of about $52 \mathrm{~nm}$ from the edge of the superlens. This small distance introduces difficulty when attempting to collect the information, hence translating the image to the far-field is desirable for improved imaging. 


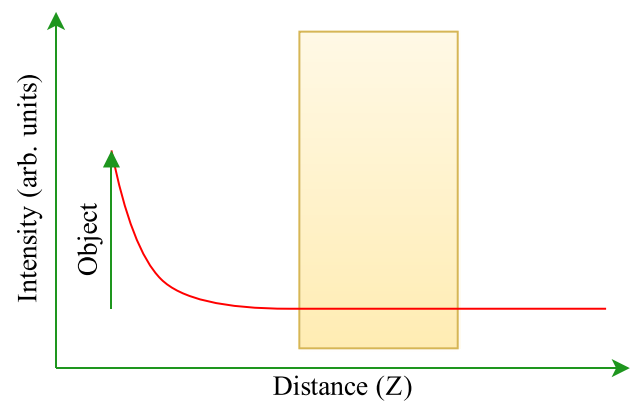

(a)

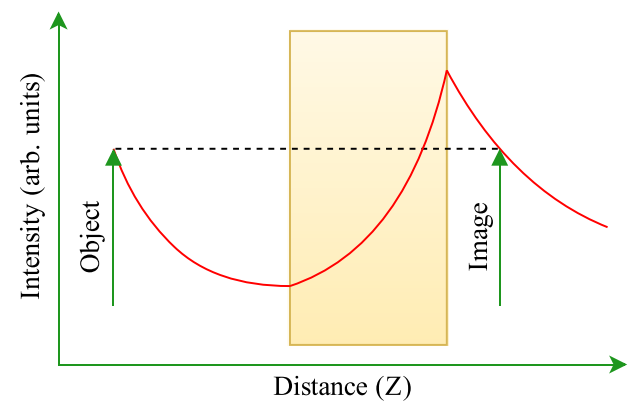

(b)

Figure 2.7: Exponentially decaying evanescent mode (red) travelling through (a) a lens with negligible plasmon resonance. The exponential decay of these modes is reversed in (b) a lens that supports SPR, allowing for an image to be realised at the image plane.

\subsection{Nano-scale diffraction grating}

With the addition of a nano-scale diffraction grating (NSDG) after the superlens, light can be intentionally scattered to 'move' the image to the farfield. Under the right conditions this can allow for sub-diffraction limited images to be reconstructed in the far-field.

The cause of this near-field to far-field transition is a result of Moiré fringes which occur from the frequency mixing of the near-field image and the NSDGs [39]. This works by translating unresolvable high spatial frequencies into low spatial frequencies by shifting incident field wave vectors, $k$ into their respective diffraction orders, i.e., $k^{\prime}=k \pm \Lambda m$, where $k^{\prime}$ is the transmitted wave vectors, $k$ is the incident wave vectors, $\Lambda=2 \pi / p$ is the grating wavenumber, $p$ is the periodicity of the gratings, and $m$ is the diffraction order. The far-field information at some $k^{\prime}$ can be considered a superposition of the various diffraction orders of each $k$ [40]. Hence, these incident wave vectors can be simultaneously mapped into a single propagating wave from the $-1,0,+1$ diffraction orders. Given sufficiently small NSDGs, the high resolution information of the object can be transmitted 
through these propagating wave orders to become detectable in the farfield [8]. These NSDGs must have a periodicity, or pitch smaller than the wavelength of the excitation light [41]. For example, if an excitation wavelength of $325 \mathrm{~nm}$ is used, the NSDG pitch should be below $300 \mathrm{~nm}$, with around $200 \mathrm{~nm}$ being an ideal trade-off between wave vector mapping efficiency and fabrication complexity. The transition theory is illustrated in Figure 2.8.

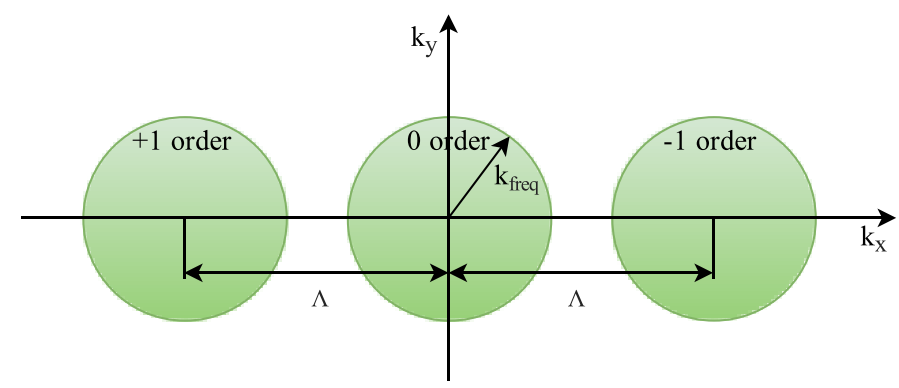

Figure 2.8: Concept of near-field to far-field transition. Typical area accessible by optical microscopes is the low spatial frequency in Fourier space defined by the central circle with radius $k_{\text {freq }}$. The outer circles represent evanescent mode frequencies higher than what is detectable, until relocation of $-1,+1$ orders to 0 order occurs.

With the addition of a NSDG to a superlens, the recovered information is able to be moved to the far-field for detection. This arrangement is known in literature as a far-field superlens (FSL) [8,39, 42-44].

\subsection{Fabrication issues, challenges, and consider- ations}

While the design of an FSL has been outlined, the films and features require a great deal of exactness and defect-free fabrication procedures. This section details extra steps which are required to overcome the inherent defects introduced by real world fabrication. 


\subsubsection{Surface roughness}

Surface roughness is a common measurement in determining the quality of a surface area or profile [45] and is one of the main quantities used to determine the quality of a film supporting SPR. A smoother film supports evanescent mode production with reduced overall loss as the mode travels along the surface [46]. Therefore, a film with a lower surface roughness will generally have improved SPR performance over a rougher film.

The roughness of a surface is quantised by the size and density of surface deviations which occur in the direction orthogonal to the true or ideal surface. These deviations, typically presented as peaks and valleys have an inherent random distribution over the surface. As a result, surface roughness must be defined by an average, $S_{a}$, or as a root-mean-squared (RMS; the average squared value of a continuously varying quantity), $S_{q}$. These two values are defined:

$$
S_{a}=\frac{1}{N} \sum_{i=1}^{N}\left|y_{i}-y_{o}\right|,
$$

and,

$$
S_{q}=\sqrt{\frac{1}{N} \sum_{i=1}^{N}\left(y_{i}-y_{o}\right)^{2}}
$$

where $N$ is the number of data points and $y_{i}$ is the vertical distance from the mean height, $y_{o}$, to the $i^{\text {th }}$ point.

Surface roughness can be attained with instruments that are able to accurately measure or determine surface topology. The most common instruments are surface profilometers, such as the Veeco Dektak 150, and atomic force microscopes (AFM). These instruments typically operate with a stylus or cantilever which contacts the surface to provide a very accurate measurement of the surface topology with ångstrom $(\AA ; 1 \mathrm{E}-10 \mathrm{~m})$ precision. Direct contact with the surface provides a very accurate reading of a sample but can easily cause damage to the surface if not operated 
correctly. An example of this measurement technique can be seen in Figure 2.9. AFM resolution is typically much greater and more reliable than the Dektak and will therefore be used as the predominant surface roughness measuring technique herein.

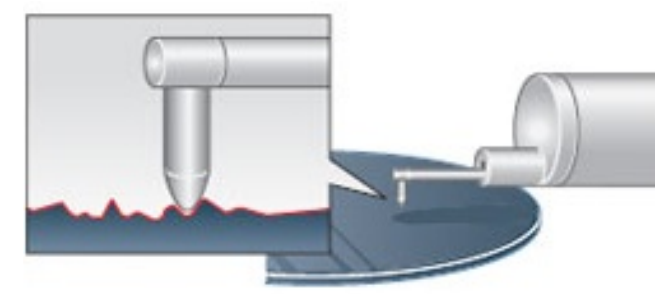

Figure 2.9: Concept of stylus operated profilometers, illustrating measurement of surface roughness [47].

\subsubsection{Grating size, shape, and roughness}

A considerable challenge in creating an FSL arises from the NSDG fabrication. Photolithography systems to expose gratings can produce undesirable results. These include the shape of the grating profile, along with the roughness of the grating. For the gratings to work optimally, they must have perfect periodic repetition and parallelism. Any deviation from this results in lower efficiency for any specific plasmonic optics application. For example this would result in higher loss of information during near-field to far-field conversion. Hence NSDG efficiency is a paramount consideration during fabrication. Line width roughness (LWR) describes the deviation of the grating from the ideal perfect straight grating. Assuming these deviations are random, produced through the exposure or development procedure, the effective uniform repetition of the gratings is deteriorated, and the grating efficiency is reduced. A lower grating efficiency will result in a further obscured reconstruction of an image at the far-field, effectively reducing tangible resolution. Figure 2.10(a) illustrates the appearance of LWR, overlaid with ideal gratings. Line-edge roughness 
(LER), the roughness of the profile of the grating is also a property to consider, but is typically less crucial in producing high quality metal gratings as deposition direction of subsequent metal films is perpendicular to LER direction.

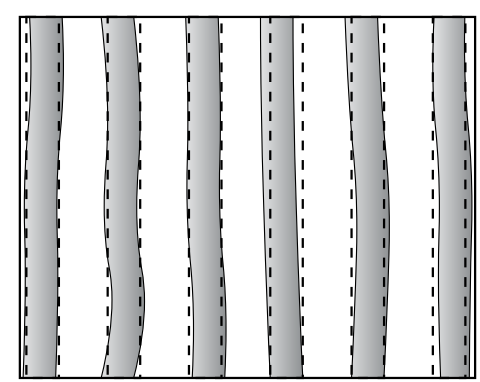

a)

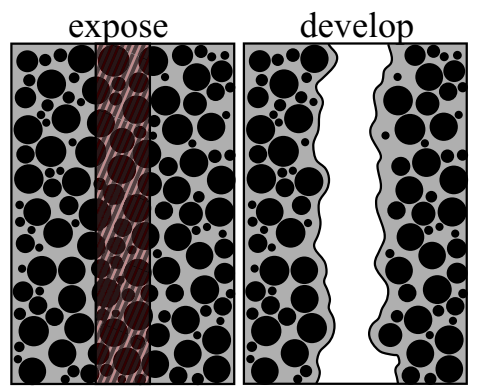

b)

Figure 2.10: (a) Exaggerated illustration of LWR compared to ideal gratings shown by dashed lines, (b) development of polymer aggregate (black circles) and surrounding polymer (grey) which causes LWR. Most of polymer aggregate that is even partially exposed is etched, resulting in a coarse line, instead of the intended red area.

LWR is inherent in photoresist at very small scale objects, such as gratings. When the gratings fabricated in photoresist approach widths of $\sim 200 \mathrm{~nm}$ LWR becomes a vital consideration to the fabrication process. It is not possible to prevent LWR, but merely to reduce it. LWR is caused mainly by photoresist itself, however random uncertainties in the fabrication process also contribute. The resist can be thought of as polymer aggregate in a sea of polymer, hence there are different sized particles of polymer in the photoresist. When these particles are exposed, even if only partially, they tend to dislodge from the surrounding polymer, resulting in roughened sidewalls [48]. An illustration of this process can be seen in Figure 2.10(b). As a result, strong emphasis on fabrication quality and possible detrimental effects during exposure and development must be investigated. 


\subsection{Literature Survey}

Extensive work has been done in regards to sub-wavelength imaging with superlenses. With some work being theoretical $[19,49,50]$, there are those who have experimentally proven the ability to reconstruct sub-wavelength images using superlenses [35,36,51], and even FSLs [42]. However, those who have achieved far-field imagery have been unsuccessful in creating a 'free' far-field superlens; that is, a lens which is not fabricated around a sample, and which may be reused on more than one sample. Furthermore, the FSLs which have been previously fabricated and experimentally tested have consisted of only one dimensional spatial relocation; that is, a one dimensional (1D) grating [49-51]. Previously reported sub-wavelength imagery could be improved upon given an FSL with a coupling element spanning over two dimensions (2D) [52-54].

\subsubsection{Surface roughness}

Additionally, research which has gone into FSLs has lacked an emphasis on the Ag film surface roughness. Kröger and Kretschmann [55] show that there is a distinct negative effect on thin film EM transmission given large surface roughness. This is further supported in the context of Ag films by Fang, et al. [56]. That being said, some work has been done on smooth Ag films but have yet to be correctly implemented for use in superlens design $[7,35,42]$.

Stefaniuk, et al. [5] experiment with seed layers of titanium (Ti), nickel (Ni), and germanium (Ge) deposited in advance of $\mathrm{Ag}$ to improve surface roughness. These seed layers provide the subsequent $\mathrm{Ag}$ films with improved deposition conditions, often resulting in much smoother films. Of the three seed layers, Ge provides surface roughness down to $\sim 0.6 \mathrm{~nm}_{\mathrm{RMS}}, \mathrm{Ni}$ $\sim 0.9 \mathrm{~nm}_{\mathrm{RMS}}$, and $\mathrm{Ti} \sim 2.0 \mathrm{~nm}_{\mathrm{RMS}}$. From here, the specific resistivity of each seed layer is measured to find $\mathrm{Ni}$ has the best overall surface roughness and specific resistivity when matched with Ag. However, the undesirably 
high resistivity of Ag matched Ge can be reduced. Chen, et al. [57] show that a thermal anneal at specific temperatures and duration can greatly reduce resistivity by approximately $70 \%$, to similar or equal levels of $\mathrm{Ni}$. Formica, et al. [6] utilise copper $(\mathrm{Cu})$ as an ultrasmooth seed layer, leading to an overall system of $S_{q}(\mathrm{Cu}+\mathrm{Ag}) \simeq 0.4 \mathrm{~nm}_{\mathrm{RMS}}$ and $\rho=8 \Omega / \mu \mathrm{m}$. The surface roughness is very low, and resistivity may reduce with a thermal anneal. Finally, silver oxide $\left(\mathrm{AgO}_{\mathrm{x}}\right)$ has been promoted for use as a seed layer to improve SP interaction for Ag films [58]. These are avenues which require more focus.

\subsubsection{NSDG Fabrication}

While NSDG fabrication has been pursued, very expensive equipment is typically required in order to achieve the small pitch needed for FSL performance. Electron-beam lithography (EBL) and focused ion beam milling (FIB) are the more common techniques used to fabricate NSDGs [39, 42, 59-62] but both are infeasible due to cost, and their extremely slow pattern writing times. EBL and FIB typically expose samples via raster scans, hence a smaller pitch or larger samples have increasing exposure times in the order of 24 hours or more. The cost and time required per sample greatly hinders the widespread implementation of EBL/FIB for NSDG fabrication and viability as a constant production source for FSLs. In contrast, IL systems can expose large areas concurrently in a few minutes, and cost is relatively low. However, an optimised exposure method is required in order to produce effective NSDGs.

In light of this, the goals of this thesis cover the increased surface smoothing of silver films in order to increase evanescent mode enhancement. Along with this, IL systems will be optimised to produce NSDGs with pitch down to $200 \mathrm{~nm}$ to move recovered imagery to the far-field for detection. 


\section{Chapter 3}

\section{Photonic structure design}

Fabrication of thin films becomes difficult at the nano-scale due to the surface roughness inherent in thin Ag films. This chapter details the procedure to overcome these issues. Furthermore, the fabrication procedure for 1D and 2D NSDGs and an overall free FSL is described.

\subsection{Silver thin film properties}

The surface roughness of $\mathrm{Ag}$ is most prominent when deposited in thin (several nanometer) layers. The deposition of Ag can produce two 'phases', which are mainly dependant on the resultant film thickness: as Ag is deposited on a substrate, it forms isolated clusters [63]. These discontinuous areas display unwanted, extraordinary absorption of plasmon resonance [64], inhibiting the evanescent wave enhancement properties which Ag typically affords. Moreover, the metal islands greatly increase the film's surface roughness [65]. As Ag is continually deposited, the islands begin to connect, producing a discontinuous metallic film. This point, is known as the percolation threshold and occurs at around $25 \mathrm{~nm} \mathrm{Ag} \mathrm{films}$ on borosilicate. At this point the film begins to exhibit its useful optical properties [66]. As Ag is deposited past the percolation threshold, Ag fills the areas between islands, and finally produces a continuous film, resulting in much lower surface roughness. 


\subsubsection{Seed layers}

Seed layers act as surrogate substrates, which lie between the substrate and $\mathrm{Ag}$, and can provide improved conditions for $\mathrm{Ag}$ to be deposited on compared to bare substrate. For example, Ge offers high surface energy, 1.3-1.835 $\mathrm{Jm}^{-2}$, much higher than common substrates such as silicon oxide $\left(\mathrm{SiO}_{2}\right)$ and borosilicate glass at $0.26 \mathrm{Jm}^{-2}[67,68]$. The higher surface energy affords $\mathrm{Ag}$ highly increased wetting as its atom clusters, which when deposited on $\mathrm{Ge}$, tend to be flatter than the same clusters deposited without the initial Ge wetting. This produces a smoother overall surface [69] and has shown surface roughness reduction by up to 70\% [70]. A smoother surface should reduce the disruption of optical light transmission and should also produce a system with less loss.

This is generally the case [71], however, very thin seed layers produce high optical loss in the film system due to the quantum confinement effect [72]. Therefore, one must aim to not only produce a smooth film, but also highly efficient film systems such that losses are kept to a minimum. Chen, et al. [57] furthered seed layer smoothing, and produced promising results by an intert gas annealing technique of $\mathrm{Ge} / \mathrm{Ag}$ films at $\sim 300^{\circ} \mathrm{C}$. Annealing Ag films typically develops high surface roughness caused by omni-directional grain growth [73]; however, thin Ge seed layers tend to restrict $z$-axis growth of $\mathrm{Ag}$ during an anneal. An anneal of an $\mathrm{Ag}$ film atop a Ge seed layer results in a primarily lateral grain growth. This is ideal as it is thought that grain boundaries cause a certain degree of scattering from the result of very slight interfacial differences in refractive index [74]. A larger average grain size implies less grain boundaries and hence less loss. Therefore a thermal anneal of a Ge/Ag film system could greatly improve optical transmission, while maintaining low surface roughness.

$\mathrm{Cu}$ is proposed to wet $\mathrm{Ag}$ films in a similar manner with an even higher surface energy of 1.96-2.24 $\mathrm{Jm}^{-2}$ [75]. Cu seed layers used by Formica, et al. [6] show a significant reduction of Ag surface roughness down to $S_{q}=0.4 \mathrm{~nm}$. 


\subsubsection{Silver oxide seed layer}

Silver oxide $\left(\mathrm{AgO}_{\mathrm{x}}\right)$ has been promoted in literature for its properties which allow extraordinary effects when used to produce nano-scale features [76], and thin films to produce optically excited electronic structures [77]. Furthermore, $\mathrm{AgO}_{\mathrm{x}}$ has some interesting photonic enhancement abilities which can be implemented as photonic waveguides and high density data storage [78]. Finally, Martin, et al. [79] have used $\mathrm{AgO}_{x}$ to create novel isolated $\mathrm{Ag}$ features through the thermal decomposition into $\mathrm{Ag}$ and $\mathrm{O}_{2}$ which is not possible through other typical fabrication procedures. Through this mechanism, a reduction of surface roughness of the isolated Ag features was observed, leading to the possibility of its extension to thin film smoothing. Given the specialised effects of $\mathrm{AgO}_{x}$ in nano-scale $\mathrm{Ag}$ features and its photonic effects, it is a good option to consider for use as a seed layer.

Crystallography considerations are paramount when it comes to the $\mathrm{AgO}_{\mathrm{x}}$ seed. Due to its oxide nature, $\mathrm{AgO}_{\mathrm{x}}$ can form various structures during deposition, if not coerced to form in one main structure and direction. When $\mathrm{AgO}_{x}$ is deposited onto a substrate, it typically orientates itself with respect to the crystal structure of the substrate; however, for an FSL to perform optimally, the main substrate on which all materials are built must be non-birefringent, i.e. non-crystalline.

Birefringent substrates effectively supply different polarisations of incident EM fields with distinct refractive indicies. Crystals, and materials with crystal structures are inherently birefringent. Hence, substrates used for plasmonic optics should ideally be amorphous. An amorphous substrate has no dominant crystal direction, and so an oxide seed layer cannot form preferentially upon it. A material, such as $\mathrm{AgO}_{\mathrm{x}}$, will then form random directions during deposition, causing stress in the film. Stressed films can go on to form dislocations, producing hills, valleys, and defects in general which increases surface roughness and reduces film quality [80].

One method to mitigate stress in the film is to deposit extremely slowly, on the order of $0.05 \AA / \mathrm{s}$. This slow deposition rate should allow for each 
continuous area of film to relax before new layers are added, reducing overall film surface roughness. Furthermore a post-growth anneal may help to reduce the stress from the film, thus smoothing the surface $[81,82]$.

\subsection{Grating fabrication process}

The design and fabrication details of creating periodic gratings for the use in plasmonic optics are presented below. This section outlines photoresist grating fabrication steps, along with metalisation of these gratings. Furthermore, small feature sizes in the nano-scale are desired and the additional techniques for their optimised fabrication is discussed.

\subsubsection{Photoresist}

Ag gratings are constructed from photoresist templates, which allow a stencil, known as a mask, to be 'copied' onto a substrate. Photoresist must be applied onto a substrate though use of a spin coater in order to attain a uniform thickness over the entire substrate. Photoresist thickness can be controlled through both the speed at which it is spun onto a substrate and the duration of the spin, but the thickness is also dependant on the viscosity of the photoresist itself. A common photoresist, AZ 1518, was initially chosen due to its availability, ease of development, and its wide use in literature [83].

\subsubsection{Mask aligner}

Typical photoresist exposure methods utilise mask aligners. These operate by allowing a mask to be positioned over a sample, covering areas of interest. Once the mask is aligned, ultraviolet (UV) light is shone onto the uncovered areas for a set exposure time. The mask aligner at VUW is a Karl Suss MJB3 mask aligner and can attain minimum feature sizes 
of around 1-2 $\mu \mathrm{m}$, but in order to produce gratings which will be useful for the creation sub-diffraction limited images, the pitch must be reduced ten-fold.

Moving to smaller pitch gratings naturally offers less area between subsequent gratings for light to travel between. Eventually, in the many hundreds of nanometers, the wavelength of light becomes an issue (the same issue this thesis aims to overcome) and prevents sufficient light to pass through the mask producing under-exposed samples, producing low resolution and low fidelity samples, or unwanted results in general. This can be overcome by sophisticated illuminations system for advanced mask aligner lithography using Köhler integrators, illuminations filter plates, and fourier lenses [84], however these are excessively complex. Furthermore, mask aligners require the sample to be placed a specific distance from the mask itself. This variable distance, which can be hard to maintain across many samples, can reduce resolution of resultant photoresist profiles as seen in Figure 3.1. Therefore, as gratings pitch reduces below $2 \mu \mathrm{m}$, the mask aligner system will be replaced with an interference lithography (IL) setup.

\begin{tabular}{|c|c|c|c|c|}
\hline Contact & $20 \mu \mathrm{m}$ & $50 \mu \mathrm{m}$ & $100 \mu \mathrm{m}$ & $200 \mu \mathrm{m}$ \\
\hline & & & \\
\hline & & & & \\
\hline
\end{tabular}

Figure 3.1: Photoresist profiles from mask aligner simulation illustrating separation between sample and mask in contact mode $(0 \mu \mathrm{m}), 20 \mu \mathrm{m}$, $50 \mu \mathrm{m}, 100 \mu \mathrm{m}$, and $200 \mu \mathrm{m}$. Red is exposed resist profile, and black outline is intended mask profile [85]. 


\subsubsection{Interference lithography}

An IL system essentially functions in the same way as the mask aligner, exposing specific parts of the photoresist. A helium-cadmium (He-Cd) laser with a wavelength of $325 \mathrm{~nm}$ is reflected through a focusing lens and then projected through a pinhole $(5-15 \mu \mathrm{m})$ which acts as a low pass filter to attain a Gaussian profile, effectively removing noise from the laser beam. The light is then shone onto both the sample and a Lloyd's mirror, where interference patterns are created from the mirrors reflection producing nodes and anti-nodes on the sample, resulting in a grating profile, as illustrated in Figure 3.2.

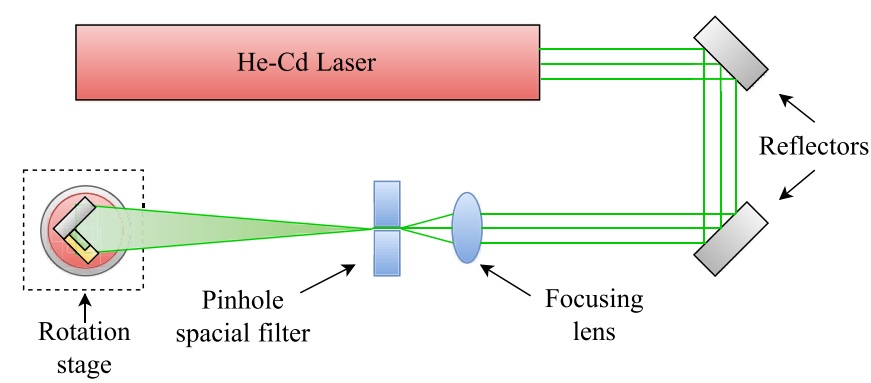

(a)

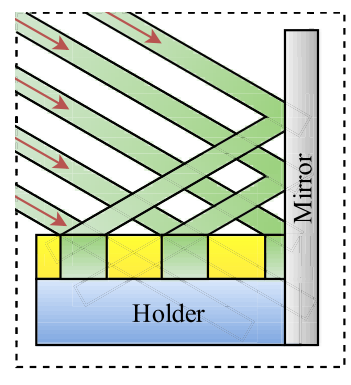

(b)

Figure 3.2: IL system illustrating (a) placement, focusing, and filtering of laser light for sample exposure, (b) detail of rotation stage and Lloyd's mirror.

The IL system has its advantages over the mask aligner, as it can immediately produce features with a $1 \mu \mathrm{m}$ separation. By altering the angle of the mirror and sample, which move together normal to each other, to incident laser light, it is also possible to increase or reduce this separation. By changing the angle of incidence, the feature sizes change by Equation 3.1 [86].

$$
p=\frac{\lambda}{2 \sin \theta},
$$


where $p$ is the interference pattern period, $\lambda$ is the laser wavelength and $\theta$ the rotation stage's angle of incidence to the laser light. For example, a linear $1 \mathrm{D}$ grating with a period of $1 \mu \mathrm{m}$ requires $\theta$ of $\sin ^{-1} \frac{\lambda}{2 p}=9.35^{\circ}$, given $\lambda=325 \mathrm{~nm}$.

The angle dictates the pitch of the gratings, but we must also have repeatable methods in determining and supplying a 'dose' of light to the sample. The dose, $d$ can be calculated given the power density $\left(I_{\text {mirror }}\right)$ of the interference pattern $\left(I_{\text {pattern }}\right)$. This is dependant on the power density of the light which exposes the sample $\left(I_{\text {sample }}\right)$. These terms are implicitly derived from the schematic setup, which can be seen in Figure 3.3.

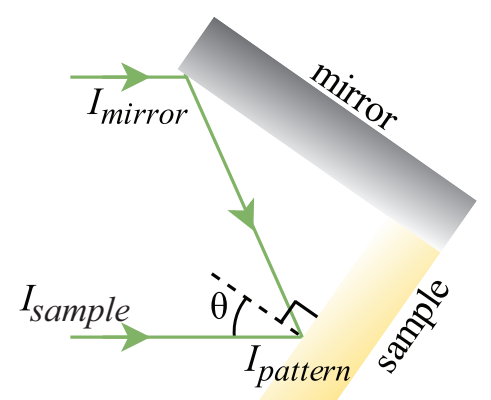

Figure 3.3: Illustration of terms used in IL dosage calculations derived from impinging light on Lloyd's mirror stage.

Using a Thor Labs S120VC 120-1100 nm power sensor connected to a PM100A power meter placed immediately in front of the sample holder, it is possible to measure the power to the sample, $P_{\text {sesnor }} \sim 0.200 \mathrm{~mW}$ (this value is not constant between power cycles of the laser and is therefore measured before each exposure onto samples). The power measured by the sensor must be normalised per area. Given the aperture diameter of 
the sensor is $9.5 \mathrm{~mm}$ [87], the area, $A$, is

$$
\begin{aligned}
A & =\pi \times\left(\frac{\text { diameter }}{2}\right)^{2} \\
& =0.709 \mathrm{~cm}^{2} .
\end{aligned}
$$

Hence, the power density at the sensor, $I_{\text {sensor }}$, is

$$
\begin{aligned}
I_{\text {sensor }} & =P_{\text {sensor }} / A \\
& =0.200 \mathrm{~mW} / 0.709 \mathrm{~cm}^{2} \\
& =0.282 \mathrm{~mW} / \mathrm{cm}^{2}
\end{aligned}
$$

and the density incident on the sample, $I_{\text {sample }}$, is

$$
\begin{aligned}
I_{\text {sample }} & =I_{\text {sensor }} \times \cos \theta \\
& =0.278 \mathrm{~mW} / \mathrm{cm}^{2},
\end{aligned}
$$

where $\theta=9.35^{\circ}$. Furthermore, the density of the beam reflected on the sample from the mirror, $I_{\text {mirror }}$, is

$$
\begin{aligned}
I_{\text {mirror }} & =I_{\text {sensor }} \times \cos \theta \times R_{\text {mirror }} \\
& =0.207 \mathrm{~mW} / \mathrm{cm}^{2},
\end{aligned}
$$

where $R_{\text {mirror }}$ describes the reflectivity of the mirror at $\lambda=325 \mathrm{~nm}$, given approximately 0.97 reflectivity. This gives the peak interference pattern density of

$$
\begin{aligned}
I_{\text {pattern }} & =\left(\sqrt{I_{\text {sample }}}+\sqrt{I_{\text {mirror }}}\right)^{2} \\
& =1.099 \mathrm{~mW} / \mathrm{cm}^{2} .
\end{aligned}
$$

Finally, from the pattern density, the dose may be calculated by integrating over the time, $t$, for which the sample is exposed:

$$
\begin{aligned}
d & =\int_{0}^{t} I_{\text {pattern }} \cdot \mathrm{d} t \\
& =I_{\text {pattern }} \times t .
\end{aligned}
$$


Therefore, given Equations 3.3-3.7, typical doses of $150-300 \mathrm{~mJ} / \mathrm{cm}^{2}$ require exposure times from 30-60 seconds.

IL has much higher resolution when compared with the mask aligner, but has its own limits. From Equation 3.1 along with the He-Cd $325 \mathrm{~nm}$ laser, the period is limited between $165-3000 \mathrm{~nm}$.

\subsubsection{Development}

Development of the photoresist is required after exposure to etch the photopattern into the resist. Wet development is commonly used as the development technique. By submerging a pre-exposed sample in developer for a set amount of time, the desired gratings can be created. The sample must then be submerged in a stop solution, typically de-ionised water $\left(\mathrm{DiH}_{2} \mathrm{O}\right)$, to prevent over-development.

Developing photoresist is an iterative process, requiring many 'sacrificial' samples to locate the optimum point of development. Developing too long results in very thin gratings, along with very rounded gratings, making subsequent metalised lift off much more challenging, whereas developing for too short a time effectively results in a continuous film of photoresist with grating indentations in it. These two extremes can be seen in Figure 3.4.

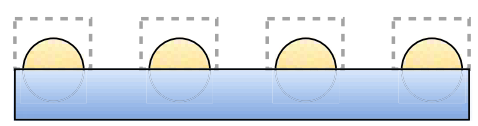

a)

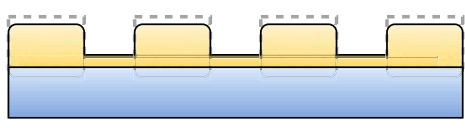

b)

Figure 3.4: Comparison of a) over-developed and b) under-developed photoresist profiles. Dashed gratings are ideal, yellow gratings are resultant photoresist gratings.

The case where development is insufficient will always lead to gratings which cannot be metalised. Without having etched through the photoresist to the substrate, a metal layer would exist entirely on the photoresist, 
and lift off, where the photoresist is removed, would remove all metal, leaving a bare substrate. This is a challenging aspect of the development process as it is extremely difficult to differentiate between each of the developing extremes at the macro-scale. Therefore, the iterative process must be scrutinised under scanning electron microscope (SEM) and AFM.

\subsubsection{Image reversal photoresist}

Positive photoresist, like AZ 1518, is often very troublesome to work with at very small feature sizes around $1 \mu \mathrm{m}$. It tends to produce rounded and shallow profiles, rather than a hard edge. This makes the fabrication of square features, such as gratings, extremely difficult to properly manufacture, and often produces unusable samples.

Image reversal photoresist (IRP) is used in a similar fashion, where areas are exposed and developed, but with the addition of another exposure step: one must expose areas which are desired, then the sample is baked, crosslinking the exposed areas, producing inert photoresist. Another exposure, referred to as a 'flood' exposure is then performed over the entire sample. The flood exposure produces soluble photoresist where it was not initially exposed and baked. The sample is then developed, retaining the baked areas. An illustration of this process can be seen in Figure 3.5.

One of the main advantages of IRP is its inherent undercut in the photoresist profile. This is illustrated in Figure 3.5 where the final photoresist gratings are shaped as inverted trapezoids, rather than squares (from the idealised profile of positive photoresist). This undercut is extremely useful when it comes to adding metal layers. The resultant shapes of IRP and positive photoresist offer a critical distinction when a metal layer is added over the photoresist. The profile which positive photoresist develops allows a metal layer continuity over the entire surface of the sample, whereas IRP causes discontinuous sections between gratings, which are left over after lift-off, and are hence ideal. An example of this can be seen in Figure 3.6. 


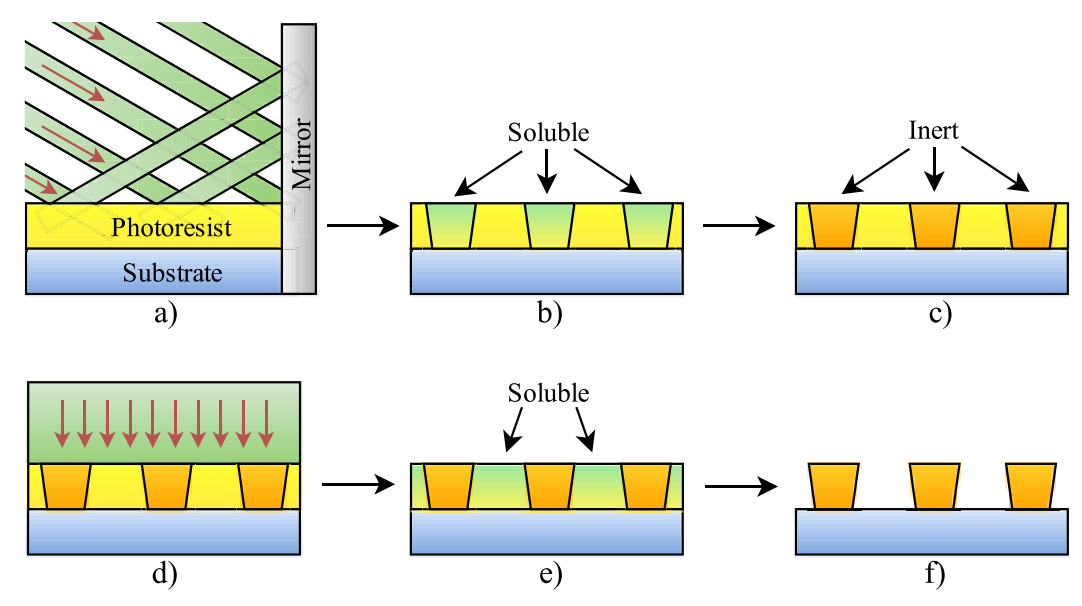

Figure 3.5: Simplified image reversal steps: a) expose areas to keep, b) these areas become soluble, c) high temperature bake leaves soluble areas inert, d) flood exposure over entire sample, e) these newly exposed areas become soluble, while previously exposed areas remain inert, d) development removes only the newly soluble photoresist.

It should be noted that negative photoresist produces a similar undercut profile; however, it has much lower resolution than IRP, reaching a limit at approximately $1 \mu \mathrm{m}$. Furthermore, negative photoresists often require carcinogenic solvents for development [88].

While the development process for IRP is almost identical to that of positive photoresist, the initial structure of the IRP gratings offers greater robustness to over-development. This is due to the undercut of each grating, which reduces the effect of rounded profiles. Each extreme of IRP development can be seen in Figure 3.7. It is possible to see that while the gratings in Figure 3.7(a) are very over-developed, resulting in incorrect period and height, liftoff would still be possible, producing functional metal gratings. This added robustness of grating over-development-through the use of IRP-aids in the fabrication and testing of FSL design. 


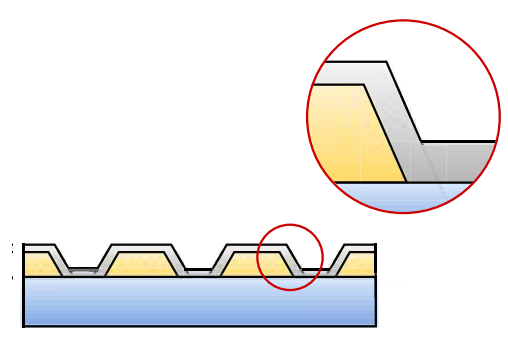

a)

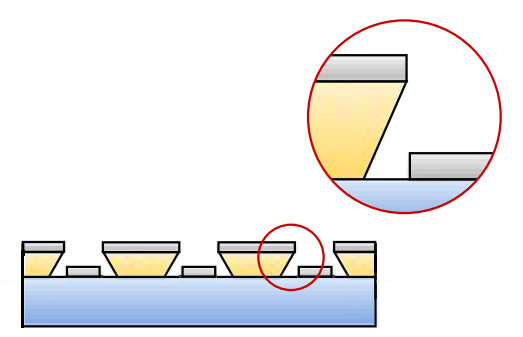

b)

Figure 3.6: Comparison of a) positive photoresist profile and b) IRP profile after metalisation. Insets illustrate continuous and discontinuous metalisation, respectively.

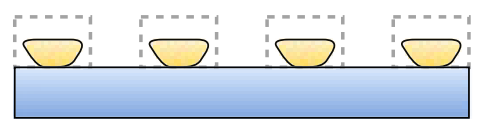

a)

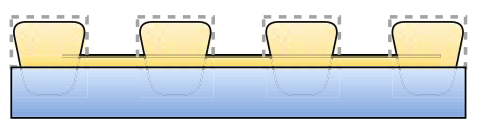

b)

Figure 3.7: Comparison of a) over-developed and b) under-developed IRP photoresist profiles. Dashed gratings are ideal, yellow gratings, resultant photoresist gratings, and blue is the substrate.

\subsubsection{Grating quality and optimisation}

The profile of the grating is a useful quantifiable property of the 'goodness' of the gratings, but it should not be the sole quantity on which grating quality is based. From a top-down view, it is possible to see the lengthwise direction of the gratings, and their line-width roughness (LWR). LWR is typically reduced using complex methodologies, such as nano-imprint lithography [89], the design of tailored polymer photoresist [90], and extreme ultra-violet (EUV) exposure [91], where photoresist design and EUVbased techniques typically require expensive equipment. However, due to the low amount of LWR we expect ( $<10 \%$ of feature width), a simpler technique can be employed. A post-exposure bake (PEB), or resist reflow process (RRP), at a temperature above the glass transition temper- 
ature of the resist will result in smoothing of the resist, heavily reducing LWR. These temperatures are typically around $100{ }^{\circ} \mathrm{C}$, with the bake lasting from 60-300 seconds. The RRP is of course dependant on the photoresist, its thickness, and dilution, but more importantly it becomes a tradeoff between the amount of rounding, and LWR. As the resist reflows, it does so in all directions causing the profile to round as LWR reduces, as illustrated in Figure 3.8. The rounding of the grating profile is detrimental causing issues with lift-off. Hence, this RRP trade-off will have to be maximised for LWR smoothing, while maintaining a sufficient profile edge to allow for lift-off.

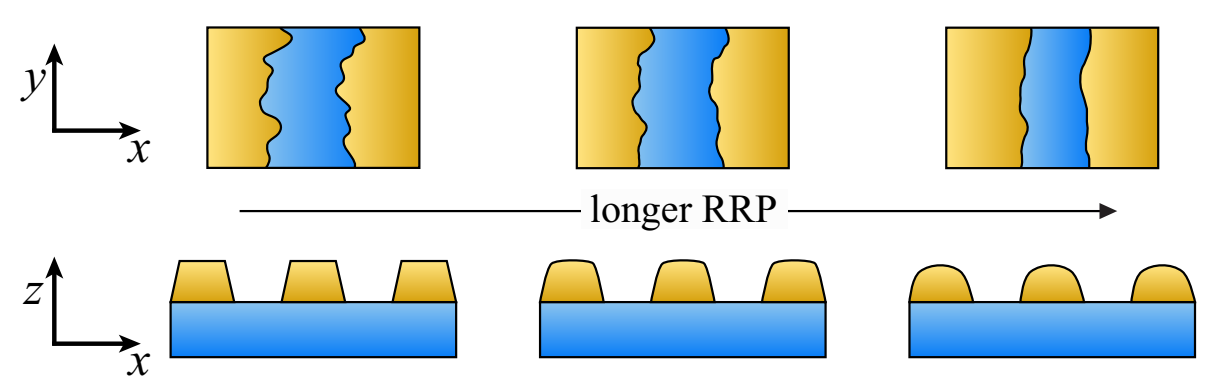

Figure 3.8: Increased RRP illustrates desired rounding on top-down view (top) and detrimental rounding on grating profile (bottom).

\subsection{Far field superlens design}

In order to more easily exploit the superlens design, the image must be reconstructed at the far-field. A far field superlens is made up of a metal film and metal gratings. These two components require extra steps in order to assemble the final FSL. Using a bottom-up approach, resulting in \{object/substrate/metal film/gratings/capping layer\} will be more straightforward to fabricate, improving fabrication yield. This is compared to a more complex top-down approach, \{substrate/gratings/metal film/capping 
layer/object\}. In order to achieve maximum evanescent enhancement, the Ag film must be fabricated such that an object can be placed as close to the FSL as possible. This restricts the thick $500 \mu \mathrm{m}$ substrate to encounter incoming light after the evanescent mode conversion to propagating waves, which the top-down approach offers. This makes the FSL more difficult to construct, but will result in much better image reconstruction due to the larger initial evanescent mode conversion. It should be noted that superlens and FSL designs which have been proposed in the past $[35,42]$ have utilised a bottom-up approach with samples mounted within the substrate region. While this results in slightly reduced evanescent mode loss, fabrication difficulty is increased, but more importantly, the sample cannot be changed as it built directly into the FSL. The method which will be used herein, and the less complex but lossy method are compared in Figure 3.9, illustrating crucial losses which can easily be introduced.

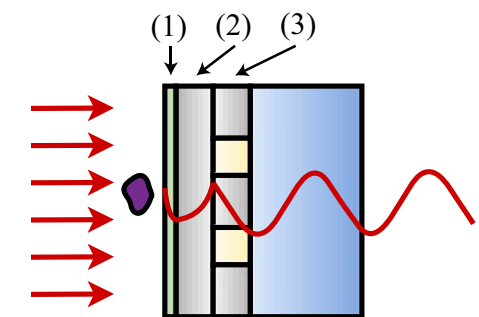

(a)

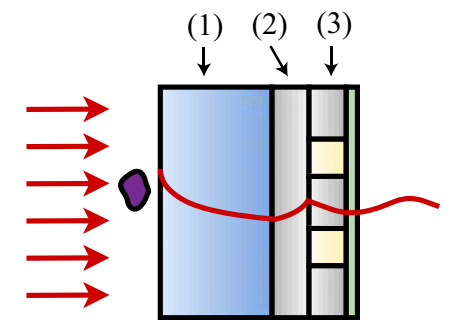

(b)

Figure 3.9: Comparison of FSL designs where (a) is complex, but provides superior small feature information enhancement, and (b) more straightforward fabrication, but results in much higher losses due to substrate placement (blue). Area (1) shows evanescent decay, (2) shows enhancement, and (3) near- to far-field translation to produce propagating waves. Red lines indicate incident light, evanescent modes, and propagating waves. Purple represents a small object, green capping layers, silver represents Ag gratings and films, and yellow PMMA filling.

The more difficult FSL fabrication method requires an Ag film to be de- 
posited on top of metal gratings. For this, we must first fill in the spaces between the metal gratings with an inert dielectric such that losses are kept to a minimum. Polymethyl methacrylate (PMMA) is an optically 'inert' substance. It is highly transparent with a refractive index of about 1.5 at optical wavelengths [92]. This means EM waves which pass through PMMA experience little loss, and more importantly, little alteration to phase of each polarisation. This results in almost unaffected optical information, which is a necessity for use in superlens design.

By filling the space between the gratings with PMMA and then removing excess PMMA, a smooth and level pseudo-substrate can be formed above the gratings. This planarisation step is crucial in providing an ideal scaffold for the Ag film to be deposited on. Once a metal film is deposited, a thin capping layer can be added. This is a protection layer to prevent oxidisation of the metal layers, and mechanical damage of the metal film. The FSL fabrication process is outlined in Figure 3.10.

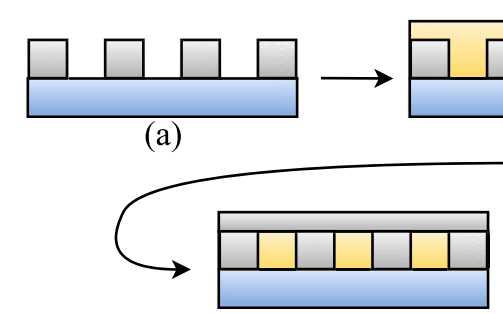

(d)

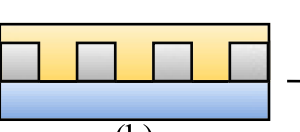

(b)
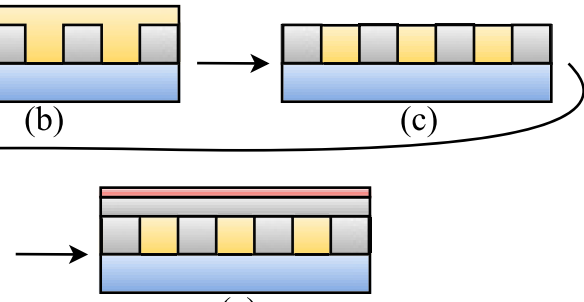

(e)

Figure 3.10: Simplified fabrication steps for the FSL: (a) create metal gratings on substrate, (b) add PMMA backfill, (c) planarise PMMA, (d) addition of Ag film, and (e) capping layer. 


\section{Chapter 4}

\section{Plasmonic film fabrication}

This chapter details the experimental procedure devised from design and theory highlighted in Chapters 2 and 3 for an Ag superlens. Furthermore, results are given for each optimised design along with discussions and explanations on their niche properties and characteristics.

\subsection{Thin film fabrication}

Metal films were deposited on 3" diameter 20/10 scratch-dig dual-side polish (DSP) Schott Borofloat 33 wafers (UniversityWafer) after which these were cut to $10 \times 10 \mathrm{~mm}$ samples. The borosilicate substrates were cleaned to remove contamination and improve film quality and uniformity. This was achieved with multiple sonicated solvent rinses in acetone and isopropyl alcohol (IPA), drying with pressurised nitrogen $(99.99 \%)\left(\mathrm{N}_{2}\right)$, baking at $100^{\circ} \mathrm{C}$ for 2 minutes, and finally cleaning using $50 \mathrm{~W} \mathrm{O}_{2}$ plasma for 1 minute. Film stacks containing $\mathrm{AgO}_{\mathrm{x}}$ seed layers were fabricated using radio frequency (RF) magnetron sputtering (HHV Auto500), with the layers deposited sequentially under base pressures of about $2.0 \times 10^{-6} \mathrm{mBar}^{1}$ and deposition pressures of $1.7 \times 10^{-2} \mathrm{mBar}$. Flow rates of 6 standard cu-

\footnotetext{
${ }^{1} 1 \mathrm{mBar}=100 \mathrm{~Pa} \approx 0.75$ Torr
} 
bic centimetres per minute (sccm) of argon and $4 \mathrm{sccm}$ of oxygen were used for $\mathrm{AgO}_{\mathrm{x}}$ seed layers, and only $6 \mathrm{sccm}$ of argon for $\mathrm{Ag}$ films. All other films were fabricated using thermal evaporation (Angstrom Engineering Nexdep 200) at base pressures of about $4.5 \times 10^{-6} \mathrm{mBar}$. Deposition rates were monitored using quartz crystal oscillators, hence all thicknesses quoted herein are mass average thicknesses, rigorously calibrated through atomic force microscope (AFM) (Nanosurf NaioAFM) in contact mode, surface profilometer (Dektak 150), and scanning electron microscope (SEM) (Jeol 6610LV) measurements. Deposition rates, purity, and supplier for each deposition material are shown in Table 4.1. Depositions were performed at room temperature $\left(20^{\circ} \mathrm{C}\right)$, although a small increase of about $10^{\circ} \mathrm{C}$ was observed due to the thermal evaporation and sputtering processes.

Table 4.1: Purity, supplier, and deposition rates for each deposition material used herein.

\begin{tabular}{llll}
\hline Material & Purity $(\%)$ & Supplier & Dep. Rate $(\AA / s)$ \\
\hline Ag sputter target & 99.99 & Plasmaterials & $0.25(\mathrm{Ag})$ \\
& & & $0.08\left(\mathrm{AgO}_{\mathrm{x}}\right)$ \\
Ag pellets & 99.99 & Kurt J. Lesker Company & 0.30 \\
Ge pellets & 99.999 & Kurt J. Lesker Company & 0.08 \\
Cu pellets & 99.999 & Kurt J. Lesker Company & 0.08 \\
\hline
\end{tabular}

\subsection{Ge seed layer}

Initial control samples fabricated without a seed layer prior to deposition produced very rough Ag surfaces of $>1.4 \mathrm{~nm}_{\mathrm{RMS}}$ when measured using the AFM. Figure 4.1 visually illustrates the roughness of Ag without a seed, and with a $1 \mathrm{~nm}$ Ge seed, emphasising the substantial effect a Ge seed layer has on the overall surface roughness of an Ag film. 


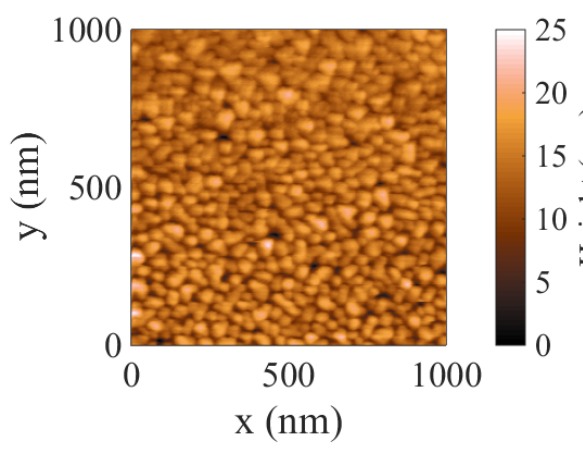

(a)

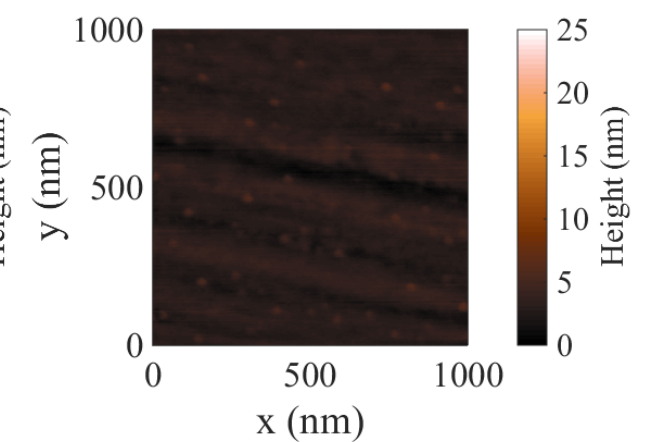

(b)

Figure 4.1: Comparison of AFM profile scans of (a) a $10 \mathrm{~nm}$ Ag film on borosilicate $\left(\mathrm{S}_{\mathrm{q}}=2.68 \mathrm{~nm}_{\mathrm{RMS}}\right)$, and (b) $1 \mathrm{~nm} \mathrm{Ge} / 10 \mathrm{~nm} \mathrm{Ag}$ film on borosilicate $\left(\mathrm{S}_{\mathrm{q}}=0.64 \mathrm{~nm}_{\mathrm{RMS}}\right)$ illustrating high roughness of Ag with no seed layer.

Figure 4.1(a) shows the Ag islands which occur before the percolation threshold, the point at which isolated islands begin to interconnect [93], is reached. They are isolated from each other and their diameter is relatively large, leading to the large surface roughness. This is in contrast to the smooth continuous film which the Ge/Ag films provide in Figure 4.1(b). It is possible to see an immediate surface roughness reduction upon the introduction of the Ge seed layer. Surface roughness decreases to $\sim 0.6 \mathrm{~nm}_{\text {RMS }}$ upon the addition of a $1 \mathrm{~nm}$ Ge film in advance of an $\mathrm{Ag}$ films. Furthermore, Figure 4.2(a) shows the smoothing effects of a Ge seed layer as the subsequent Ag film thickness is varied. Uncertainties for each data point are calculated standard errors from at least 12 measurements from 3 samples. Without a seed layer, we have observed via SEM imaging that $\mathrm{Ag}$ percolates somewhere between $20-30 \mathrm{~nm}$, with continuous films being produced at $\geq 35 \mathrm{~nm}$. The smoother Ge/Ag films, along with the downward $S_{q}$ trend with increasing Ag thickness is indicative of Ag's reduced percolation threshold to $<10 \mathrm{~nm}$ thick films on Ge seed layers, resulting in an increasingly smoother film system as Ag is deposited up to $40 \mathrm{~nm}$. From this decrease in surface roughness, it is possible to conduct 
a thermal anneal of the Ge/Ag films to reduce optical losses as discussed in Section 3.1.1. Optical transmission measurements were recorded for increasing annealing temperatures, and can be seen in Figure 4.2(b). Annealing was performed using a highly insulated thermal annealing tube furnace with pure argon (99.99\%) flowing through the furnace for $180 \mathrm{~min}$ utes before each 3 minute anneal.
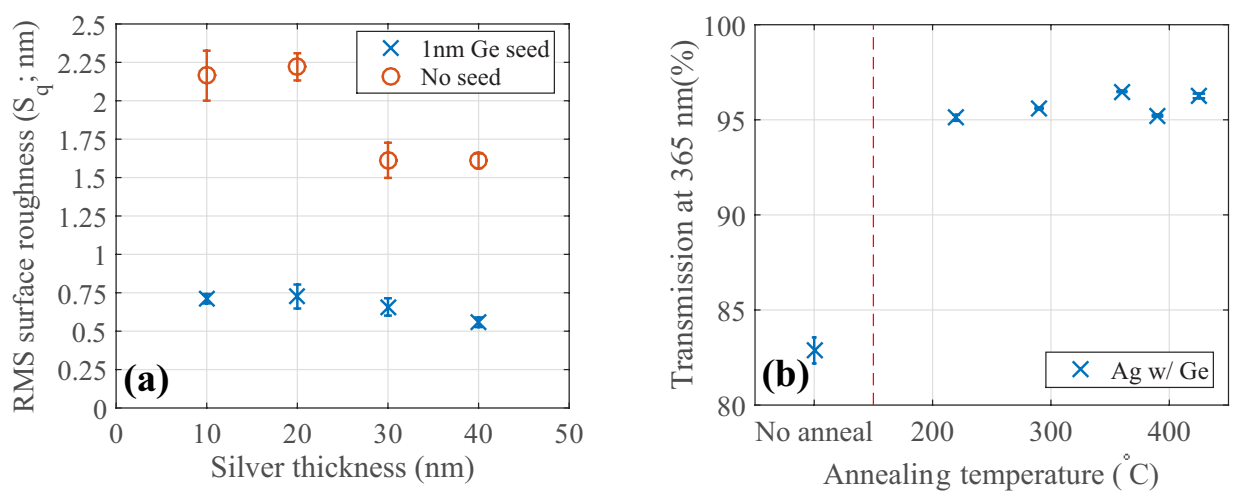

Figure 4.2: (a) Ag surface roughness varying with thickness and Ge seed layer, (b) $1 \mathrm{~nm} \mathrm{Ge/10} \mathrm{nm} \mathrm{Ag} \mathrm{film} \mathrm{optical} \mathrm{transmission} \mathrm{with} \mathrm{annealing}$ temperature.

In theory, a Ge seed layer restricts Ag grain growth to the lateral plane, hence we would not expect surface roughness to increase during a thermal anneal of Ge/Ag film system. The results shown in Figure 4.3 confirms this, showing that surface roughness in fact decreases as the Ge-seeded film is annealed at increasing temperatures. Furthermore, $1 \mathrm{~nm} \mathrm{Ge} / 40 \mathrm{~nm}$ Ag films are compared to illustrate the diminishing effect of the percolation threshold of $\mathrm{Ag}$ after an anneal above $300^{\circ} \mathrm{C}$ regardless of thickness up to $40 \mathrm{~nm}$. For the optimum optical transmission of $96 \%$, achieved at an annealing temperature of $360^{\circ} \mathrm{C}$, the surface roughness decreases by $16 \%$ to $0.615 \mathrm{~nm}_{\text {RMS }}$. These results, supported by those in [57], suggest that any Ag film grown on a Ge seed layer for use in a plasmonic optical device should be subjected to a thermal anneal to produce optimum surface roughness and transmission, and therefore an optimum plasmonic response with this arrangement. 


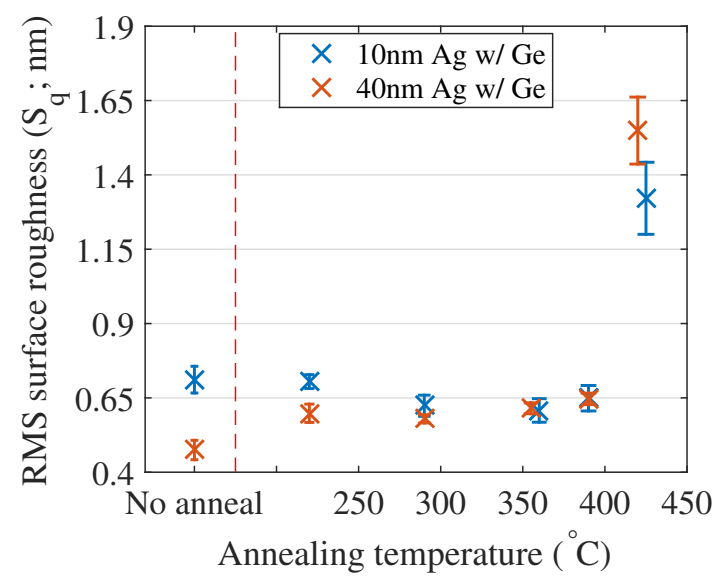

Figure 4.3: Roughness of Ge/Ag films with increasing annealing temperature for 10 and $40 \mathrm{~nm}$ thick $\mathrm{Ag}$ films.

\subsection{Cu seed layer}

Formica et al. [6] found a $\mathrm{Cu}$ seed layer resulted in low surface roughness for $6 \mathrm{~nm}$ thick Ag films; we have grown such films and obtained similar results achieving $0.6 \mathrm{~nm}_{\mathrm{RMS}}$, compared to Formica's $0.4 \mathrm{~nm}_{\mathrm{RMS}}$, with discrepancies attributed to their extra plasma etching steps, along with much lower deposition pressures. Ag grown on $\mathrm{Cu}$ seed layers was characterised using the same method as Ge/Ag films. Figure 4.4(a) shows surface roughness results, while the resultant $\mathrm{Ag}$ films from a $\mathrm{Cu}$ seed layer can be seen in Figures 4.4(b) and (d).

The smoothing effect of $\mathrm{Cu}$ is notable from the somewhat continuous $\mathrm{Ag}$ film it produces as shown in Figures 4.4(b) and (d). While much better than the isolated islands in which Ag grows with no seed layer, the $\mathrm{Cu}$ seed only smooths these $\mathrm{Ag}$ islands such that some adjacent islands are connected, resulting in continuous, but distinct paths. This is compared to the Ge seed layer where all neighbouring Ag islands have become interconnected, resulting in a continuous film, as shown in Figures 4.4(c) and (e). This is a subtle, but distinct difference between the two seed lay- 

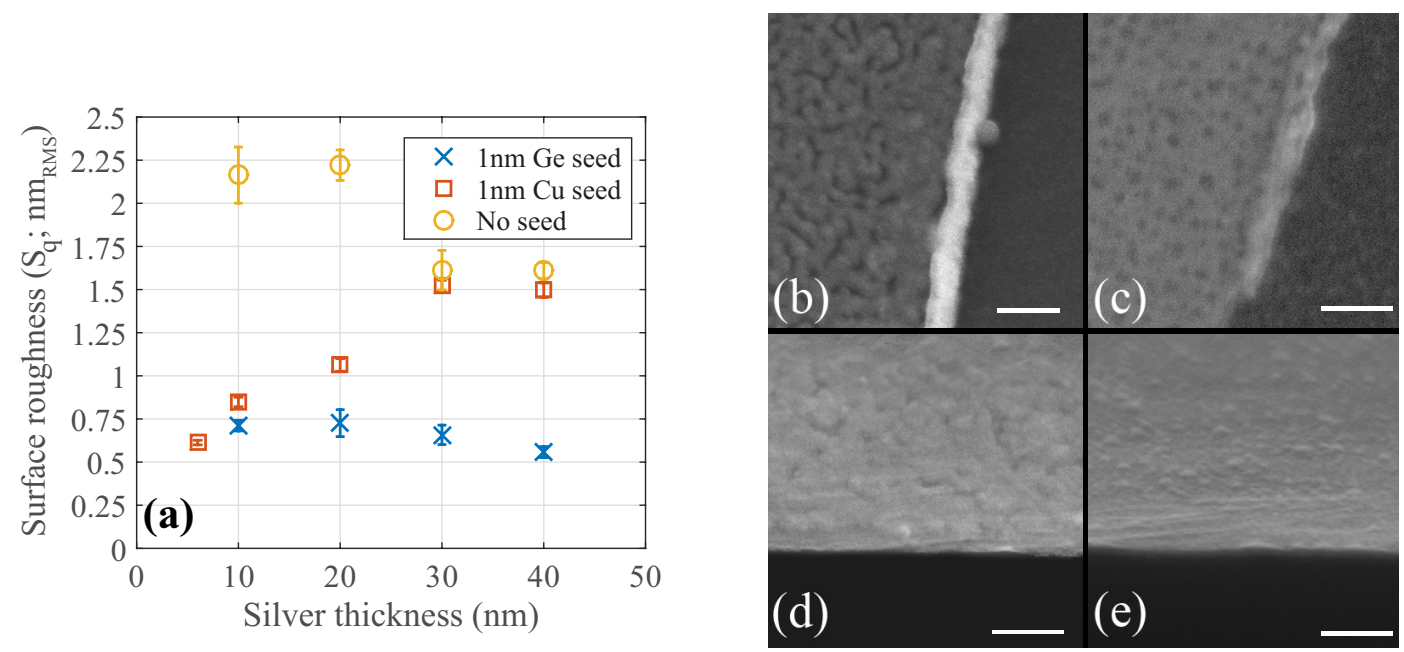

Figure 4.4: (a) surface roughness varying with thickness for films grown on $\mathrm{Ge}$ and $\mathrm{Cu}$ seed layers, as well as directly on the substrate. SEM surface (top) and profile (bottom) views of $1 \mathrm{~nm} \mathrm{Cu} \mathrm{(b)} \mathrm{\&} \mathrm{(d)} \mathrm{and} \mathrm{Ge} \mathrm{(c)} \mathrm{\&} \mathrm{(e)} \mathrm{seed}$ layers under a $10 \mathrm{~nm} \mathrm{Ag} \mathrm{film.} \mathrm{Right} \mathrm{side} \mathrm{of} \mathrm{(b)} \mathrm{\&} \mathrm{(c)} \mathrm{is} \mathrm{removed} \mathrm{film} \mathrm{to}$ show bare substrate. Lower boundary of (d) \& (e) shows edge overhang of sample. Scale bars are $100 \mathrm{~nm}$.

ers, accounting for the difference in surface roughness for $10 \mathrm{~nm}$ thick Ag films. As a result, $\mathrm{Cu}$ as a seed layer produces slightly rougher subsequent Ag films when compared to Ge, especially as Ag films approach $40 \mathrm{~nm}$ thick.

However, Ag layers around $10 \mathrm{~nm}$ thick are ideal for improved evanescent enhancement [94] - the thickness where the Cu seed falls short when compared to Ge. While the $\mathrm{Cu}$ seed layer reduces Ag roughness, in the best case from 2.16 to $0.61 \mathrm{~nm}_{\mathrm{RMS}}$, the seed seems to have low lateral influence over Ag film growth; that is, the $\mathrm{Cu}$ seed can produce smooth Ag films in thin layers, but as the Ag thickness increases, the smoothing effect of $\mathrm{Cu}$ diminishes. This returns to the mechanism with which $\mathrm{Cu}$ smooths subsequent Ag films. The high $\mathrm{Cu}-\mathrm{Ag}$ bond-dissociation energy (40.7 kcal [95]) results in more tightly packed atoms-and hence a smoother surface-at an interface compared with Ag-Ag bonds (37.6 kcal [95]). How- 
ever, as $\mathrm{Ag}$ thickness increases to orders of magnitude larger than the $\mathrm{Cu}$ seed layer, the bond energy becomes less influential towards surface topography. The Ag-Ag bonds become more dominant consequentially increasing surface roughness. These results indicate a $\mathrm{Cu}$ seed layer is both thickness-restricted and less effective in reducing surface roughness when directly compared with Ge. The resultant lack of smoothing can be seen visually by means of SEM images of $10 \mathrm{~nm} \mathrm{Ag}$ films fabricated on $\mathrm{Cu}$ and Ge seed layers, respectively, as seen in Figures 4.4(b)-(e).

In an effort to further reduce surface roughness, an annealing treatment for $\mathrm{Cu}$ was also explored. Lee, et al. [96] found rapid thermal annealing to be the optimum annealing treatment, compared to vacuum annealing and thermal nitriding, in producing a low resistivity $\mathrm{Cu}$ film, and hence a low surface roughness film [97-99]. Therefore, the thermal annealing treatment conducted on $\mathrm{Ge} / \mathrm{Ag}$ was repeated for $\mathrm{Cu} / \mathrm{Ag}$ films. As seen in Figure 4.5(a), a thermal anneal develops an increase in surface roughness for $\mathrm{Cu}$ films.
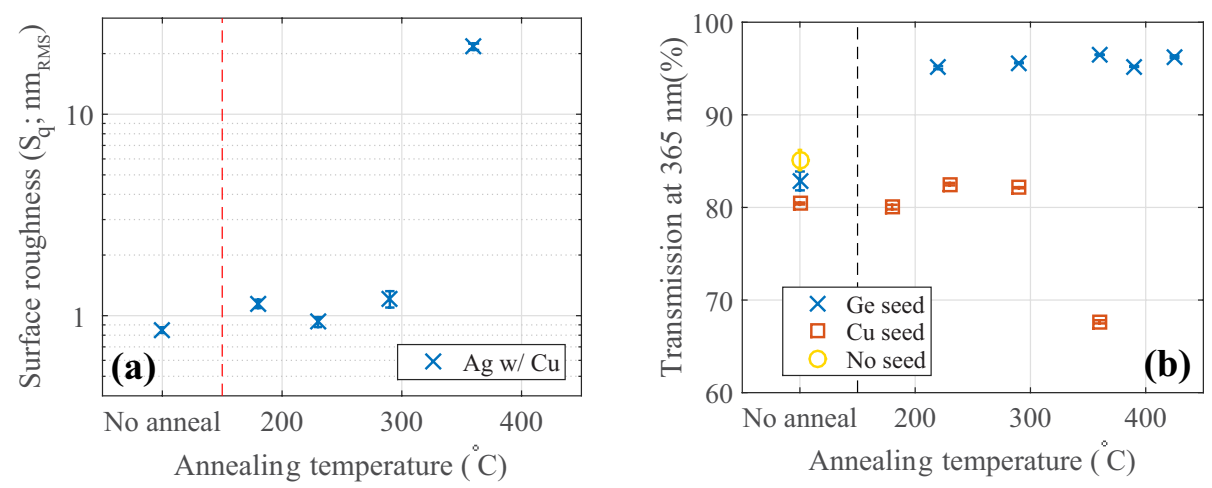

Figure 4.5: (a) Surface roughness $1 \mathrm{~nm} \mathrm{Cu} / 10 \mathrm{~nm}$ Ag films with increasing annealing temperature which results in increasing roughness. (b) Ag film transmission varying with annealing temperature and seed layer.

The roughness of films grown on $\mathrm{Cu}$ compared to Ge-seeded Ag further increases during thermal annealing. Yet, for the $200-300^{\circ} \mathrm{C}$ annealing range, the increased roughness results in a slight increase in optical trans- 
mission, as seen in Figure 4.5(b). Optical transmission in the UV range should in theory reduce with the surface roughness of thin films [100] due to introduced fluctuations in surface polarisation and magnetisation densities, leading to disruptions in transmission uniformity [55]. However, it seems an optically enhancing mechanism increases the transmission over the annealing range even with the increased surface roughness. Notwithstanding, when the optical transmission over the entire annealing range is compared to Ag with Ge seed, or even Ag alone, the high loss of $\mathrm{Cu}$ is noticeable.

These results suggest that although $\mathrm{Cu}$ does not restrict the Ag grain growth during a thermal anneal as efficiently as a Ge seed layer. Ag will tend to grow in all directions unless otherwise impeded [101,102], and so grows perpendicular to the surface (along with lateral growth), increasing surface roughness. The surface roughness trend seen in Figure 4.4(a) suggests the Ag grain growth restriction of $\mathrm{Cu}$ is limited to $<10 \mathrm{~nm} \mathrm{Ag}$ films. It should also be noted that $\mathrm{Cu}$ seed layers appear to have a low thermal resilience, producing very high surface roughness and diminished optical transmission at temperatures just over $300^{\circ} \mathrm{C}$, compared to the much higher $>450^{\circ} \mathrm{C}$ thermal threshold which Ge affords.

\section{$4.4 \quad \mathrm{AgO}_{x}$ seed layer}

Finally, $\mathrm{AgO}_{x}$ was promoted for use as a smoothing seed layer for $\mathrm{Ag}$ [58]; however, sputtering techniques to produce smooth $\mathrm{AgO}_{\mathrm{x}}$ on borosilicate substrates proved very difficult. After optimising the deposition technique for $\mathrm{AgO}_{\mathrm{x}}$ and $\mathrm{Ag}$, the final surface roughness was reduced from about $7 \mathrm{~nm}_{\mathrm{RMS}}$ to $1.75 \mathrm{~nm}_{\mathrm{RMS}}$.

Throughout the experimental procedure, the $\mathrm{AgO}_{\mathrm{x}} / \mathrm{Ag}$ interface produced very large Ag grains, and hence high roughness. Larger Ag grains are desirable in a $\mathrm{Ge} / \mathrm{Ag}$ stack as this grain growth is limited to the lateral plane due to inherent Ge properties. This results in less grain boundaries and flatter grains, both of which contribute to a smoother surface. 
However, in an $\mathrm{AgO}_{\mathrm{x}} / \mathrm{Ag}$ stack, the grains grow laterally and vertically producing much rougher surfaces, hence smaller grains are preferred.

It should be noted that the optimised $\mathrm{AgO}_{\mathrm{x}} / \mathrm{Ag}$ films were deposited sequentially in situ, via RF sputtering without breaking vacuum. This is in contrast to the other Ag films described in within which were grown by thermal evaporation. For comparison, Ag films were also deposited via thermal evaporation onto $\mathrm{RF}$ sputtered $\mathrm{AgO}_{\mathrm{x}}$ seed layers. These films were comparatively rough, with average surface roughness of $8.15 \mathrm{~nm}_{\mathrm{RMS}}$. It is unknown whether this greater roughness is due to the behaviour of the seed layer or to the contamination that results after breaking vacuum to transfer samples from the sputtering chamber to the evaporation chamber under ambient conditions. In any case, the end result was not suitable for our purposes. Thermal annealing treatments were also explored for $\mathrm{AgO}_{\mathrm{x}}$, but caused very high surface roughness on the order of $11 \mathrm{~nm}_{\mathrm{RMS}}$.

In summary, the $\mathrm{AgO}_{x}$ seed produces very rough films when compared to $\mathrm{Ge}$ or $\mathrm{Cu}$ seeds. $\mathrm{AgO}_{\mathrm{x}}$ and Ge seed layers are directly compared in Figures 4.6(b) and (c) to illustrate the ineffectiveness of $\mathrm{AgO}_{\mathrm{x}}$ as a smoothing seed layer. Furthermore, Figures 4.6(a) and (b) demonstrate the weak smoothing effect which $\mathrm{AgO}_{\mathrm{x}}$ has on an $\mathrm{Ag}$ film by way of very similar grain size and topography as an Ag film with no seed layer.

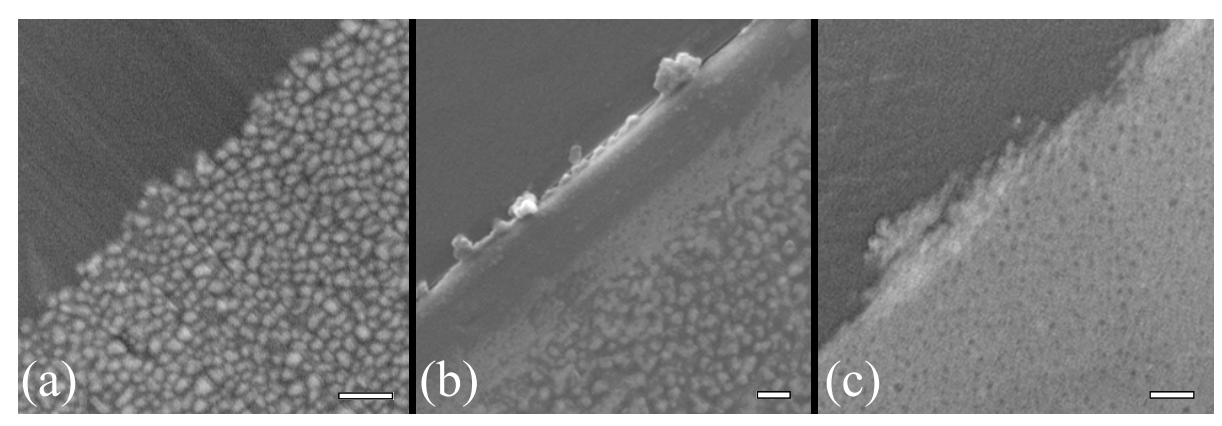

Figure 4.6: Planar SEM images of (a) $10 \mathrm{~nm} \mathrm{Ag}\left(S_{q} \approx 2.16 \mathrm{~nm}\right)$, (b) $1 \mathrm{~nm}$ $\mathrm{AgO}_{\mathrm{x}} / 10 \mathrm{~nm} \mathrm{Ag}\left(S_{q} \approx 0.85 \mathrm{~nm}\right),(\mathrm{c}) 1 \mathrm{~nm} \mathrm{Ge} / 10 \mathrm{~nm} \mathrm{Ag}\left(S_{q} \approx 0.71 \mathrm{~nm}\right)$. Top left section of each micrograph is bare substrate. Scale bars are $100 \mathrm{~nm}$. 
With the discrepancy between our results and those presented in [58], substrate effects were studied. Throughout the fabrication of thin films, we have used high quality borosilicate substrates, whereas [58] uses sapphire substrates. $\mathrm{AgO}_{\mathrm{x}}$ was deposited onto (001)-orientated sapphire substrates as a comparison to the borosilicate substrates which have been used in this study. The results presented in Figure 4.7 show a very strong reduction in surface roughness of $\mathrm{AgO}_{\mathrm{x}}$ on sapphire from 0.812 to $0.308 \mathrm{~nm}_{\mathrm{RMS}}$. This illustrates the effectiveness of oxide deposition on crystalline substrates, and explains the discrepancy between our earlier results and those published in [58]. However, we note that due to the birefringent nature of crystalline substrates, they are not suitable for all plasmonic applications. Birefringent substrates effectively supply different polarisations of incoming electromagnetic energy with distinct refractive indicies. Even with the relatively small birefringence of sapphire, $\Delta n \approx 0.0089$ [103], light separation $1 \mathrm{~mm}$ from the field's entrance to the sapphire can produce outgoing light divergence of approximately $300 \mathrm{~nm}$. This effectively reduces the resolution of any image reconstruction by obscuring the small feature information, and reduces the effectiveness of a plasmonic device itself.

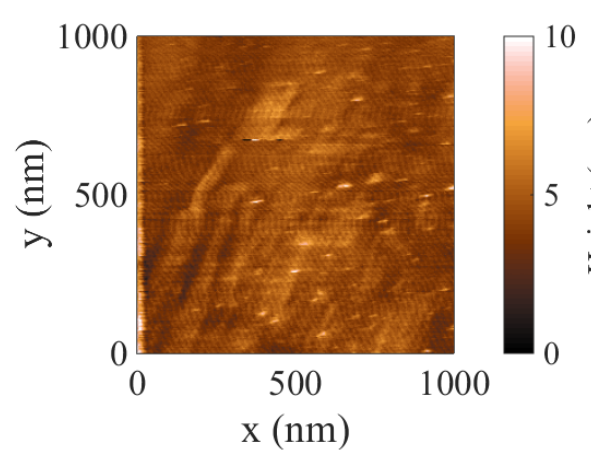

(a)

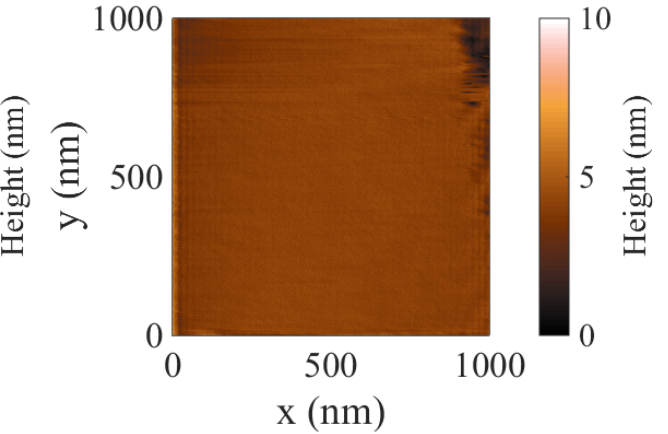

(b)

Figure 4.7: AFM micrographs of $\mathrm{AgO}_{\mathrm{x}}$ deposited on (a) borosilicate and (b) sapphire. Resultant surface roughness is $0.812 \mathrm{~nm}_{\mathrm{RMS}}$ and $0.308 \mathrm{~nm}_{\mathrm{RMS}}$, respectively. Micrographs illustrate reduced roughness of $\mathrm{AgO}_{\mathrm{x}}$ on crystalline substrates compared to amorphous ones. 


\section{Chapter 5}

\section{Grating design}

Fabrication of photoresist gratings was performed on the same $10 \times 10$ $\mathrm{mm}$ samples as detailed in Section 4.1. After the cleaning procedure, photoresist is applied using a spin coater. From here, the sample with photoresist is placed in the rotation stage of the IL system, held in place via vacuum. The sample is exposed for a time calculated from sample power and dosage. The exposed sample, if required, will then undergo a postexposure bake for 60 seconds at $100^{\circ} \mathrm{C}$. Developing the sample is done in a wet puddle of developer for a distinct development time. The sample is immediately rinsed in $\mathrm{DiH}_{2} \mathrm{O}$ for approximately twice as long as development time to stop development and to further clean the sample. Finally, the sample is dried using $\mathrm{N}_{2}$.

A requirement of the photoresist is that it must be $300-500 \mathrm{~nm}$ thick. This is the optimum thickness between achieving the best case grating profile-the longer the development, the less square the gratings becomeand having sufficient thickness to produce separation of a metalised film. Figure 5.1 shows AZ 1518 reaching a minimum thickness of approximately $2 \mu \mathrm{m}(2000 \mathrm{~nm})$ at $4000 \mathrm{RPM}$, hence standard AZ 1518 is unable to achieve this desired thickness.

AZ 1518 is a buffer solution - a weak acid and its conjugate base. As a result, its $\mathrm{pH}$ is stable with the addition of a small or even moderate 
amount of strong acid or base [104]. Hence, AZ 1518 can sustain the characteristics which are required for etching even with a dilution of select chemicals in order to reduce its minimum film thickness to the required 400-500 nm.

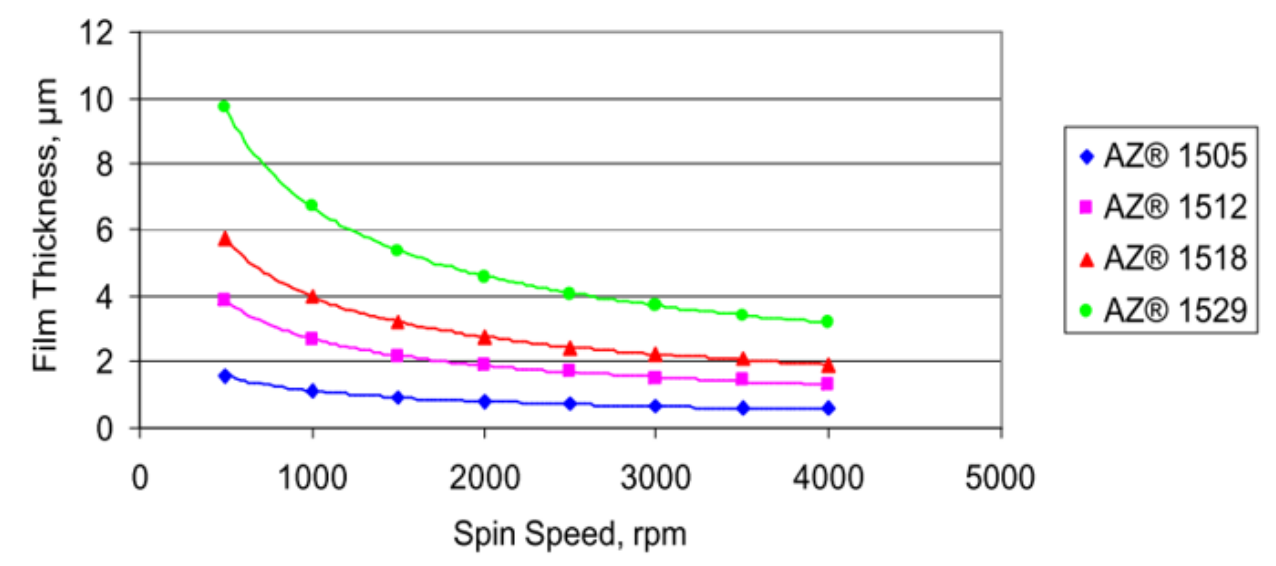

Figure 5.1: Spin speed curves for AZ 1500 resist products (6" Si wafer; soft bake $90^{\circ} \mathrm{C} / 60$ seconds) [83].

Propylene glycol monomethyl ether acetate (PGMEA) is a high purity, effective, low cost solvent designed for dilution of the AZ 15XX photoresist series. PGMEA is also an edge bead remover which removes ridges, or beads, which build up at the edge of a wafer during spin coating [105]. For these reasons, PGMEA was selected as the dilution agent, and applied to the AZ 1518 photoresist at a 1:1 ratio (by weight). The solution of AZ 1518:PGMEA was spun onto fused silica substrates at 3000 RPM for 30 seconds, and then baked at $115^{\circ} \mathrm{C}$ for 60 seconds. The resultant thickness of select samples can be seen in Table 5.1.

As per Table 5.1, it is possible to attain the desired thickness, achieving on average $409 \mathrm{~nm}$. This standard AZ 1518 photoresist is generally useful for applications with thick layers (1-10 $\mu \mathrm{m})$ and large feature sizes ( $>5 \mu \mathrm{m}$ ). Many iterations of $1 \mu \mathrm{m}$ pitch gratings in AZ1518 resulted in very rough and wavy gratings with doses of $350 \mathrm{~mJ} / \mathrm{cm}^{2}$ and 18 second 
Table 5.1: AZ 1518:PGMEA thickness results on fused silica at 3000 RPM for 30 seconds. Samples chosen at random from a batch of 20 samples.

\begin{tabular}{r|ccccc|cc} 
Sample \# & 1 & 2 & 3 & 4 & 5 & Mean & Std Dev \\
\hline Thickness (nm) & 383 & 426 & 435 & 401 & 400 & 409 & $21(5.2 \%)$
\end{tabular}

development in AZ 326 MIF developer. It was found that the photoresist itself was the main issue behind the troublesome production of gratings. The heavy dilution of AZ 1518 to achieve the required thickness greatly reduces the efficacy of its photo-sensitivity. As a result, the initial low resolution AZ 1518 is further reduced to a scale which is unusable in this application. Figure 5.2 shows gratings produced using AZ 1518 at $1 \mu \mathrm{m}$ pitch. The profile of these gratings are very triangular and show heavy LWR and LER. Metalisation and lift-off of these gratings would not produce usable metal gratings in general. At this stage, it was evident that a replacement photoresist was required in order to achieve usable NSDGs. An IRP, AZ 5214E was tested to replace the AZ 1518.

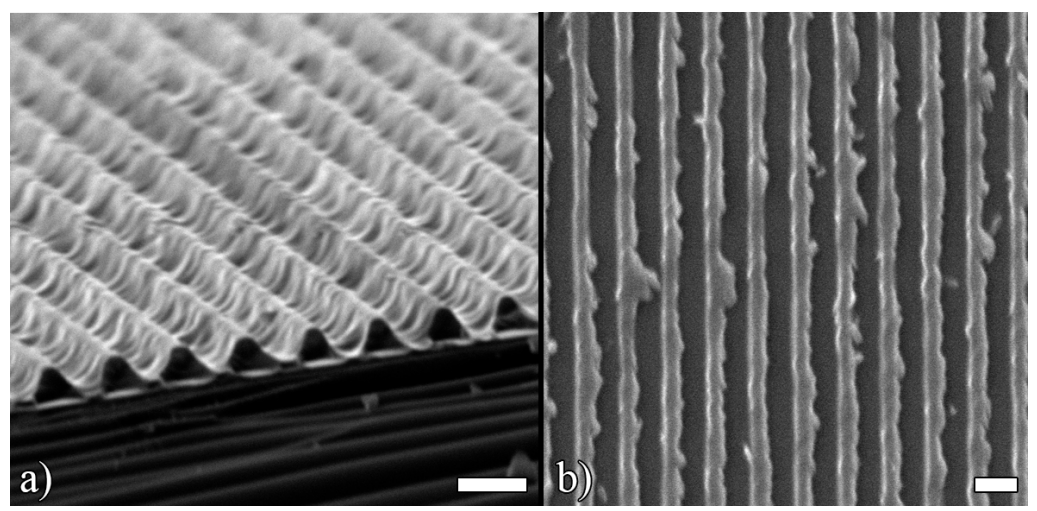

Figure 5.2: SEM images of resultant dilute AZ 1518 gratings in (a) profile, and (b) top-down. Images illustrate bad profile, and large LWR. Scale bars are $1 \mu \mathrm{m}$

Initial $1 \mu \mathrm{m}$ results with the AZ 5214E IRP seemed promising, producing much finer edge profiles than that of AZ 1518. These were created with 
an initial exposure dose of $200 \mathrm{~mJ} / \mathrm{cm}^{2}$, post-exposure bake at $100^{\circ} \mathrm{C}$ for 60 seconds, a $300 \mathrm{~mJ} / \mathrm{cm}^{2}$ flood exposure, and finally an 8 second development in AZ 726 MIF developer. However, as smaller feature sizes were attempted, similar nonidealialities as before began appearing, such as high line edge roughness both laterally and vertically. Again, AZ 5124E is not specifically made for very high resolution, thin films as required by these applications. At this point, a high resolution photoresist was required.

High resolution IRP would be the ideal case; however, there is a commercial lack of IRP which is suitable for the required thicknesses and feature sizes, so a positive high resolution photoresist was chosen. While the disadvantages of positive photoresist have been discussed herein, a very high resolution positive resist can produce sidewalls which are almost perpendicular, producing a sharp enough edge for a metal film to become discontinuous to aid in lift-off.

Using AZ 701MiR, Microchemical's highest resolution positive resist, we were able to proceed in producing small feature gratings. The AZ 701MiR had to be diluted slightly to achieve the photoresist thicknesses required. A mix of AZ 701MiR:PGMEA at 2:1 ratio (by weight) spun at 3000 RPM for 60 seconds produced $350 \mathrm{~nm}$ thick films. In a similar method to AZ 1518, the nanoscale diffraction gratings were produced at $1 \mu \mathrm{m}$ pitch with a photoresist thickness of $\sim 350 \mathrm{~nm}$. Figure 5.3 shows SEM images of all three photoresist series used herein, illustrating the effectiveness of the high resolution photoresist.

\subsection{Metalisation and lift-off}

The Ge/Ag metalisation is conducted in the same manner as for the thin film design, described in Section 4.2. After metalisation, lift-off is achieved in a wet puddle of acetone residing in a sonication bath to increase effectiveness. After sufficient lift-off duration, typically between 20-60 seconds, the sample is moved into sonicated IPA to remove metal residue, and to 


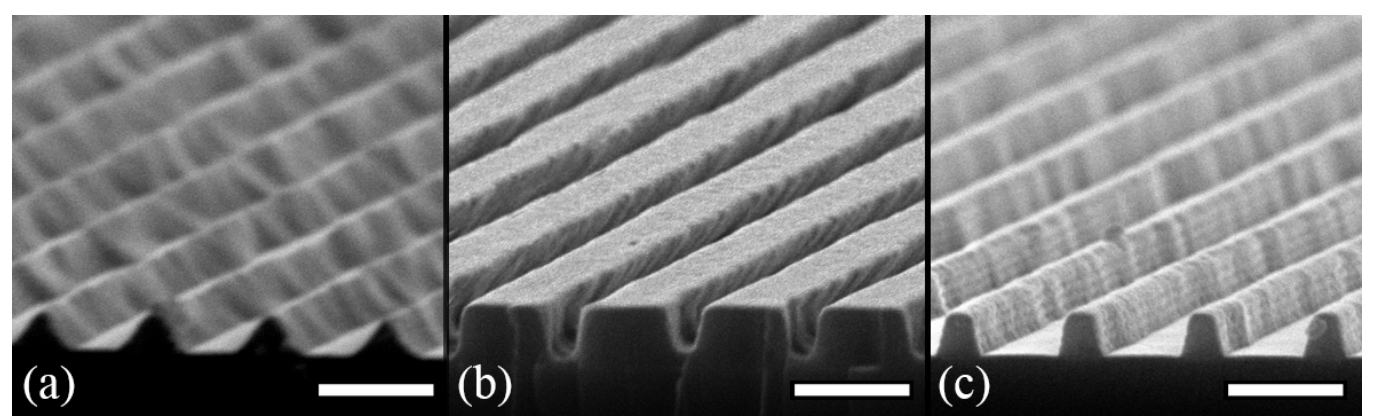

Figure 5.3: $1 \mu \mathrm{m}$ gratings fabricated using (a) AZ 1518, (b) AZ 5214E, and (c) AZ 701MiR. Photoresist performance increasing left to right. Scale bars are $1 \mu \mathrm{m}$.

clean the sample of acetone. From here, the sample is dried using $\mathrm{N}_{2}$. Utilising the optimum system from Section 4.1-a Ge/ Ag film-metalisation of the gratings is straightforward.

Lift-off was initially conducted using a wet puddle technique in sonicated acetone, and then in IPA. This technique produced sub-par results, as lift-off did not occur in large areas of samples and produced excessive amounts of metal film overhang.

These overhangs are a result of the shallow side-wall angle of the photoresist. Metalisation of these gratings results in a continuous metal film which then after lift-off, produces the undesired overhangs. As the photoresist is removed, the continuous metal film does not split at the substrate, but rather splits towards the peak of the grating, leaving free floating metal. This is shown in Figure 5.4(a), while experimental results can be seen in Figure 5.4(b).

In order to achieve maximum diffraction efficiency, and hence maximum resolvable resolution and performance from the FSL, the grating must be as ideal as possible, requiring the least amount of nonidealialities presented in Figure 5.4(b). The fabricated metal gratings adhere to the substrate over the majority of its profile, and so a more aggressive lift-off technique would improve metal edges. 


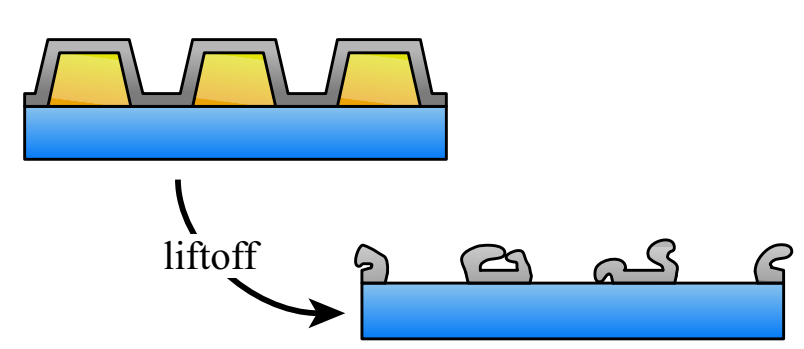

a)

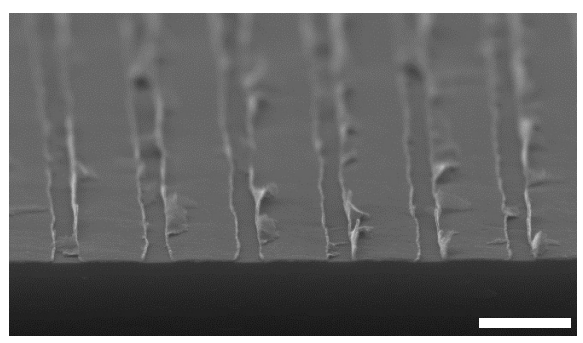

b)

Figure 5.4: (a) Illustration and (b) fabrication results of nonideal lift-off of $40 \mathrm{~nm} \mathrm{Ag}$ on borosilicate. This occurs as prelift-off metal gratings are continuous over photoresist. In (a) yellow represents photoresist, silver is $\mathrm{Ag}$, and blue is substrate. Scale bar is $1 \mu \mathrm{m}$.

In an attempt to overcome some of these issues presented through liftoff with acetone, a slightly stronger lift-off solvent was used-n-methyl-2pyrrolidone (NMP). NMP was initially disregarded due to the increased adverse health effects [106], however its superior lift-off properties necessitate its use. The lower vapour pressure yields less post-lift-off contamination, while producing improved resist removal and keeping removed particles suspended in solution more effectively [107]. This resulted in much cleaner lift-off.

Finally, lift-off was conducted in full strength sonicated NMP for 10 seconds with metalised surface upside down. As the metal particles have a higher density than the surrounding NMP, they sink away from the sample, reducing contamination. To further improve lift-off, several cleaning procedures were employed immediately after lift-off: sonicated rinses in IPA and $\mathrm{N}_{2}$ flow parallel to grating direction to both dry the sample and remove metal debris. An example of the results of this rigorous technique can be seen in Figure 5.5 where the optimum lift-off technique is compared to a simpler, lower quality technique.

With an optimised development, metalisation, and lift-off technique, it is now possible to reduce pitch in order to effectively move near field 


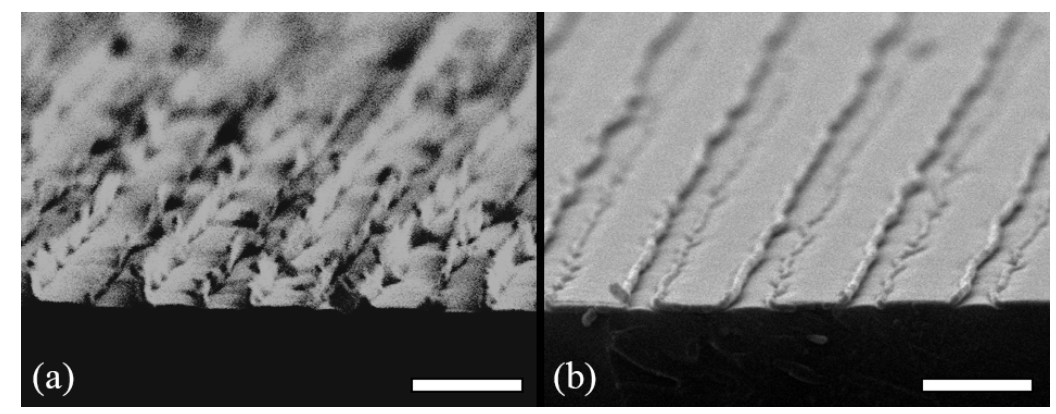

Figure 5.5: SEM micrographs of Ag gratings on borosilicate substrate. Comparison of (a) initial low performance lift-off technique producing rough Ag gratings, and (b) optimised lift-off technique resulting in much smoother Ag gratings. Scale bars are $1 \mu \mathrm{m}$.

information to the far field. As mentioned in Section 2.5, the NSDG pitch must be reduced to $\leq 200 \mathrm{~nm}$. This is possible using the IL system, but as with any process, as smaller features are required, the fabrication process necessitates more rigorous attention to detail. Furthermore, as pitch decreases, the exposure dose and development times vary, with these added variations becoming all that more pivotal towards producing functional gratings.

\subsection{Nano-scale grating idiosyncrasies}

Creating feature sizes on the micro-scale is relatively straight-forward when compared with those on the nano-scale. Very small factors which typically are not an issue at micro-scale can produce large changes in nano-scale gratings. As grating pitch should be roughly two thirds of the exposure wavelength is desired, these factors must be carefully controlled.

The main contribution factor in the fabrication of NSDGs is the substrate's interaction with incident light during an exposure. As the light passes through the photoresist, it hits the substrate and is reflected back towards incidence. The incident and reflected wave interact and produce 
standing waves within the photoresist. Typically on the micro-scale, these standing waves produce a noticeable corrugated profile in the photoresist, which does not strictly inhibit the production of micro-scale gratings, but is undesirable. Unfortunately, the standing waves occur at similar dimensions as the desired feature sizes at the nano-scale preventing the fabrication of NSDGs. A common method of overcoming these reflections is to use a bottom anti-reflective coating (BARC). BARC is applied to the substrate before the photoresist in order to effectively reduce reflections between the photoresist and substrate during exposure. The chosen BARC, AZ Barli-II 120 from Microchemicals is spun onto a cleaned substrate at $6000 \mathrm{RPM}$ for 120 seconds, and is baked at $100^{\circ} \mathrm{C}$ for approximately 120 seconds to produce a $100 \mathrm{~nm}$ layer. The photoresist layer then follows, now diluted AZ 701MiR:PGMEA 1:2 (by weight), and spun at 6000 RPM for 120 seconds to produce $92 \mathrm{~nm}$ thick layers. Figure 5.6 illustrates the considerable effect a BARC layer provides: gratings are difficult to discern in Figure 5.6(a) which does not have a BARC, but are obvious in Figure 5.6(b), which does. While the notable improvement of the gratings is easily seen, they are not ideal as of yet. Their low frequency LER waviness is also an issue, considering the gratings even intersect one another in places.

This intermingling of gratings is in fact due to local air vortices which have a time dependant nature during the exposure. These vortices interfere with the incident light and can move particulates into the beam's path causing irregular exposure patterns on samples. This manifests as the irregular beating where the peaks and troughs seen in Figure 5.6(b) produce an inhomogeneous plane, i.e. a wavy z-axis component is added. Furthermore, singular gratings at irregular intervals can become deformed and fork into two gratings, producing a coarse discontinuity within the periodic grating structure. Mathematical descriptions of these turbulent vortices are given elsewhere [108].

After the discovery of this, a light and air vortex shield was added over 


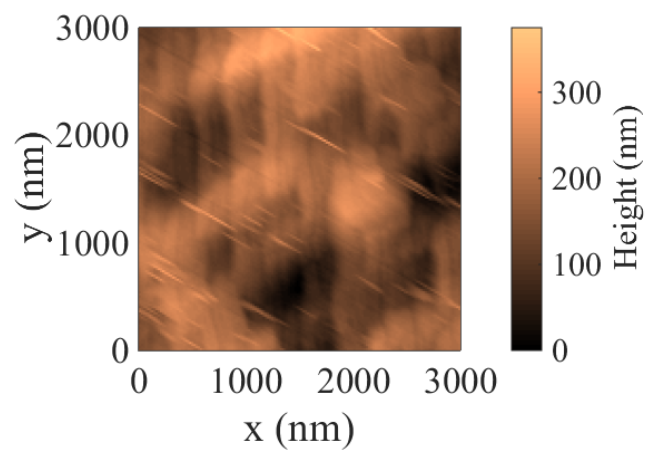

(a)

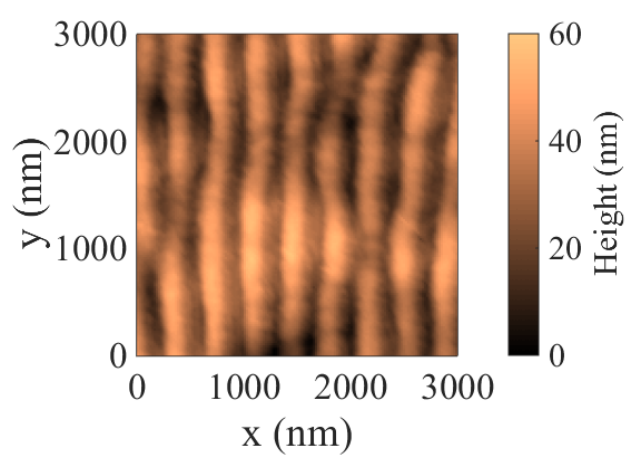

(b)

Figure 5.6: Exposure and development of $300 \mathrm{~nm}$ pitch NSDGs (a) without BARC, and (b) with BARC.

the laser exposure area in order to reduce these negative effects. Along with this, we found detrimental effects from the vacuum line holding samples in place during an exposure. Given minute long exposures, the vacuum's time dependant nature effectively shook the sample sufficiently to reduce grating quality. To prevent this, samples were mechanically held in place during exposures without vacuum. Fabrication results illustrated in Figure 5.7 show the stark contrast between samples before and after both vacuum and vortex issue rectification. While it is easy to see the increased fidelity between Figures 5.7(a) and (b), fast-fourier transforms (FFT), which describe these grating profiles in the frequency domain, also supports this improvement mathematically. The FFT of the increased development process produces much purer frequency information, shown by the large peak at $300 \mathrm{~nm}$ and reduced noise in Figure 5.7(d), when compared with Figure 5.7(c).

The detail of such features can be achieved mainly due to the BARC. While effective, it does indeed add an extra layer of complexity. Development of the upper photoresist layer produces gratings through to the BARC layer and no further. In order to produce metallised gratings, the BARC layer must be etched. This was achieved using a high power helium- 


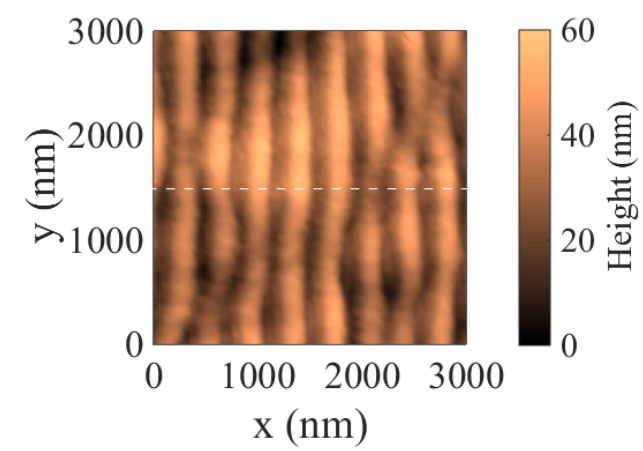

(a)

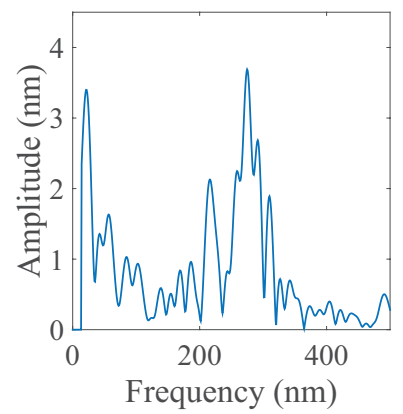

(c)

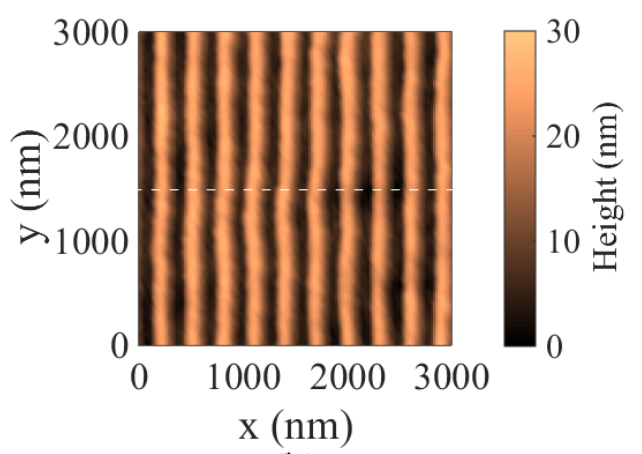

(b)

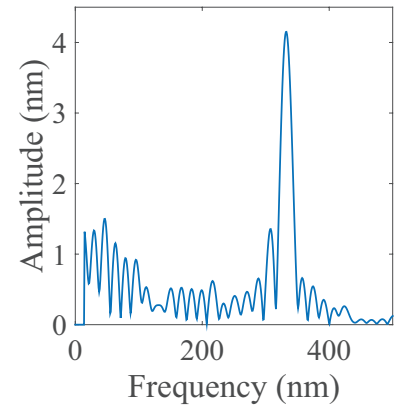

(d)

Figure 5.7: (a) $300 \mathrm{~nm}$ pitch photoresist gratings with vacuum and vortex exposure issues, (b) $300 \mathrm{~nm}$ pitch photoresist gratings with exposure issues rectified, (c) and (d) are FFTs of respective subfigures above. The FFTs in (c) and (d) are taken from single lines indicated by dashed lines in each of (a) and (b).

oxygen $\left(\mathrm{He}-\mathrm{O}_{2}\right)$ plasma in a reactive-ion etcher (RIE) [109]. RIE is a dry etching technique which uses chemically reactive plasma to remove targeted material. The plasma inside the RIE chamber is generated under vacuum using strong electrostatic fields, typically through inert gasses such as $\mathrm{He}, \mathrm{Ar}$, or $\mathrm{O}_{2}$ [110].

By using $\mathrm{He}$ and $\mathrm{O}_{2}$ input gasses at 10 and $5 \mathrm{sccm}$, respectively, with the chamber pressure at $20 \mathrm{mTorr}$ and power set to $80 \mathrm{~W}$, a plasma is generated. This plasma etches both photoresist and BARC, but the etch is more 
heavily weighted towards the BARC layer. This results in a net vertical etch through the BARC. The etch depth through the BARC can be directly controlled through the plasma etch duration and power. At $80 \mathrm{~W}$, the etch rate is approximately $44 \mathrm{~nm} / \mathrm{min}$. With $1 \mu \mathrm{m}$ gratings the $\mathrm{He}-\mathrm{O}_{2}$ etch produces BARC gratings that are approximately $30-40 \mathrm{~nm}$ high, which restricts possible metalisation thickness and hence, Ag NSDG thickness, to around $20 \mathrm{~nm}$.

Another concern with the RIE process is the isotropicity of the etch, especially in the case of nano-scale feature sizes. For example, with $1 \mu \mathrm{m}$ pitch gratings at $100 \mathrm{~nm}$ thick, we see approximately $40 \mathrm{~nm}$ isotropic lateral etch from either side of the grating, which is only a small portion of the $500 \mathrm{~nm}$ feature sizes, leaving roughly $420 \mathrm{~nm}$ wide grating peaks. However, the isotropic etch amount is constant when etching the $100 \mathrm{~nm}$ feature size gratings, resulting in a heavy lateral etch, which typically leads to premature grating removal. Isotropic etching is illustrated in Figure 5.8.

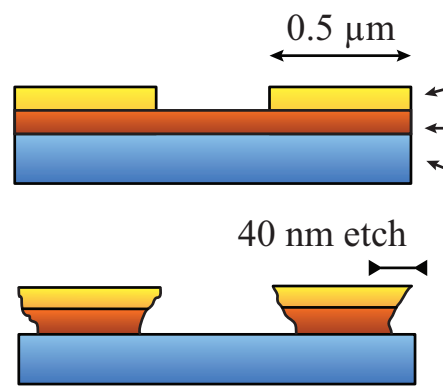

(a)

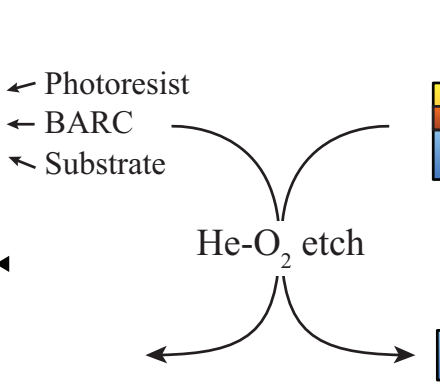

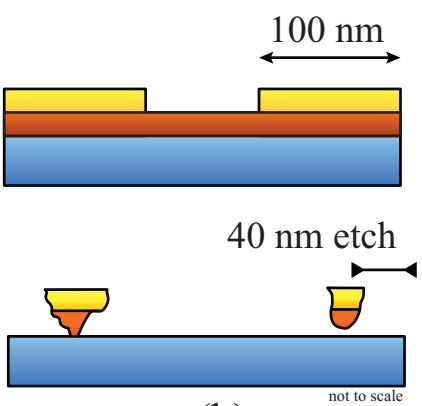

(b)

Figure 5.8: BARC etching with $\mathrm{He}-\mathrm{O}_{2}$ plasma on (a) $1 \mu \mathrm{m}$ and (b) $200 \mathrm{~nm}$ pitch. (a) shows the small isotropic lateral etch to the sidewalls of the gratings, while (b) shows excessive and catastrophic anisotropic etching due to the smaller feature size.

One solution is to decrease photoresist thickness, but a thinner photoresist layer would result in the inability to perform metalisation and lift-off at the desired Ag thickness. A photoresist layer approximately 
$20 \mathrm{~nm}$ thick would be required, which would allow for a maximum of a $10 \mathrm{~nm}$ Ag layer. However, Durant, et al. [8] show theoretically that a grating depth should be approximately 50-60 $\mathrm{nm}$ to maximise transmission through the -1 diffraction order. Ag gratings at $10 \mathrm{~nm}$ depth will only functionally transmit order -1 at $20 \%$ efficiency.

In summary, we have identified a viable process for the fabrication of an FSL. We have produced metal Ag films with surface roughness of $0.6 \mathrm{~nm}_{\mathrm{RMS}}$ and $96 \%$ optical transmission. Furthermore, the production of $200 \mathrm{~nm}$ pitch gratings was achieved using a BARC layer. However, efficiency of such an FSL is likely to be limited compared to an ideal design [8]. Optimising our process to fabricate an optimum FSL could be done with either the introduction of an oxide film between photoresist and BARC, or a more anisotropic etch, such as would be available from a deep reactive ion etcher (DRIE). Unfortunately, both of these options would require new equipment (gas extraction apparatus for safe RIE of oxide layers with fluorine-based gas, or new DRIE machine), which is currently not available. 


\section{Chapter 6}

\section{Conclusions and future work}

This thesis explores the fabrication of Ag-based photonic devices, such as superlenses and FSLs. One of the main factors contributing towards an efficient superlens being an extremely smooth surface. We have compared and discussed several seed layers for use in improving photonic performance. From the Ag thin film fabrication discussed in Section 4 we have found the Ge seed layer to provide the best initial surface roughness and optical transmission of $0.71 \mathrm{~nm}_{\text {RMS }}$ and $83 \%$, respectively. These qualities are improved upon using an inert gas thermal annealing technique in an Ar atmosphere, resulting in $0.61 \mathrm{~nm}_{\text {RMS }}$ roughness and $96 \%$ optical transmission. While Ge improves the SPR qualities of $\mathrm{Ag}, \mathrm{Cu}$ and $\mathrm{AgO}_{x}$ were less effective seed layers, resulting in rougher surfaces, or films with poor transmission at $325 \mathrm{~nm}$. That being said, we have discovered a thin $\mathrm{Cu}$ seed layer has an advantage of producing extremely smooth films with $<10 \mathrm{~nm}$ Ag thicknesses.

In addition, the plasmonic applications that we have considered requires the use of non-birefringent substrates; however, we found that $\mathrm{AgO}_{x}$ can produce very smooth films on crystalline substrates, on the order of $0.3 \mathrm{~nm}_{\mathrm{RMS}}$, which could be very useful in photonic sensors $[111,112]$ and photonic solar cells [113-115].

We also explored the fabrication of NSDGs for use in FSLs. These re- 
quired in-depth research into fabrication procedures, and extensive environmental optimisation such as the addition of bandpass light filters, $5 \mu \mathrm{m}$ pinhole Fourier filters, and air vortex shields. Chapter 5 details the different approaches to this, through the use of specialised equipment and high quality, bespoke photoresist. Through these rigorous fabrication steps, we were able to create flat, low roughness Ag gratings anywhere from 10-40 nm high. Moreover, the bounds of interference lithography were approached in the creation of high contrast sub-wavelength gratings approximately $200 \mathrm{~nm}$ in pitch.

\subsection{Future work}

\subsubsection{Film efficiency and evanescent mode production}

As previously discussed in Section 3.1.1, the addition of seed layers, especially in metal films at thicknesses around a couple of nanometers, produces high loss due to the quantum confinement effect. Nevertheless, they tend to reduce surface roughness. Currently, there is little experimental evidence which shows that this trade-off produces a net improvement to evanescent mode enhancement (EME). As a result, we have devised a simple method for detecting and comparing relative EME for Ag films with and without seed layers. Using a Kretchmann configuration [116], it would be possible to produce evanescent modes at the interface of a prism by impinging light below the critical angle, $\theta_{c}$ for total internal reflection. With this, a superlens may be fabricated on the base of the prism in an effort to enhance the produced evanescent modes. Finally, upon exiting the superlens, the evanescent modes are allowed to decay through a fluorescent dye suspended in polyvinyl alcohol (PVA). Given an incident beam at $325 \mathrm{~nm}$ from a He-Cd laser, a specific dye, 4',6-diamidino-2-phenylindole (DAPI), is excited and should produce fluorescence at $460 \pm 12 \mathrm{~nm}$. This fluoro-Krechmann design can be seen in Figure 6.1. 


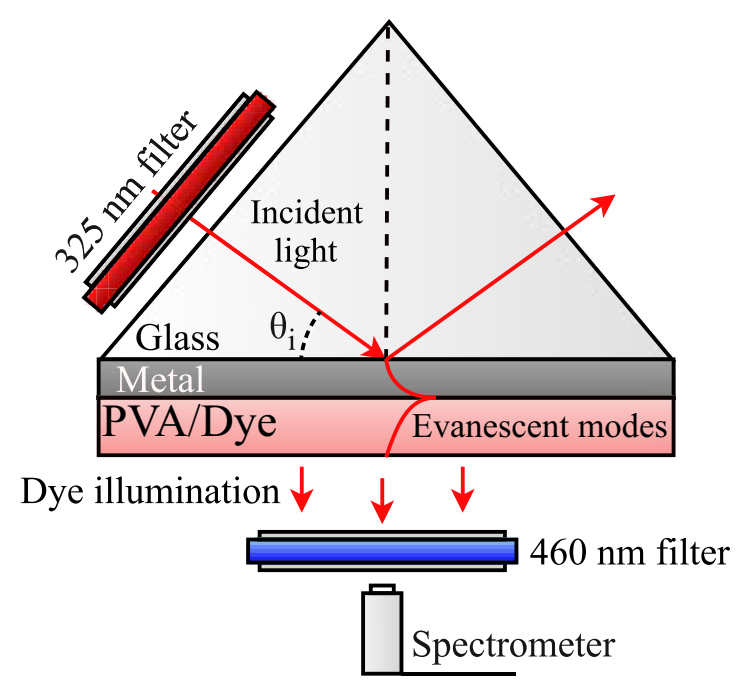

Figure 6.1: Simplified illustration of Kretchmann configuration with added dye for evanescent illumination. Red lines through the metal and PVA show evanescent enhancement intensity and decay, respectively. Incident light shows propagation direction.

The fluorescence from the DAPI could be measured easily using a spectrometer. To further reduce measured noise, a $325 \mathrm{~nm}$ bandpass filter should be placed just before incidence to the prism, and $460 \mathrm{~nm}$ bandpass filter just after the evanescent mode production. These filters both reduce the likelihood of other light sources producing excitation in the DAPI, and also reduces other wavelengths of light being incorrectly measured as $460 \mathrm{~nm}$.

As a proof of concept, we have fabricated such prisms and custom designed holders to house prisms in a thermal evaporator and spin coater. We have also produced a separate prism rotation stage. Using BF33 Schott $60^{\circ} 20 / 10$ scratch/dig prisms from Sinoptix, it was possible to evaporate smooth Ag films upon the bottom face. Figure 6.2 reports the detected spectrometer information radiated through prisms with various metal coatings of either $\mathrm{Ag}$, $\mathrm{Cu}$, or chromium ( $\mathrm{Cr}$ ).

There is obvious DAPI fluorescence detection when a $40 \mathrm{~nm}$ Ag layer 


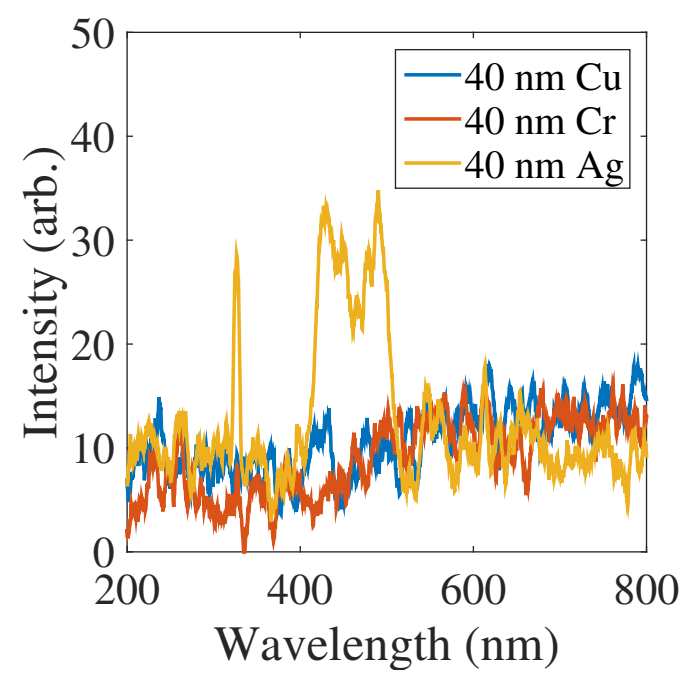

Figure 6.2: Spectrometer response from fluoro-Kretchmann setup. Measured data through Ag shows EME at $460 \mathrm{~nm}$, whereas $\mathrm{Cu}$ and $\mathrm{Cr}$ do not.

is deposited on the prism. This is compared to $40 \mathrm{~nm}$ films of $\mathrm{Cr}$ and $\mathrm{Cu}$, both of which do not show any response. We attribute this to a direct result of EME through the Ag film. The enhancement triggers the fluorescence of DAPI which is detectable by the spectrometer. This is in contrast to the negligible EME which $\mathrm{Cr}$ and $\mathrm{Cu}$ provide.

This is an important technique for measuring film quality and the EME effects which seed layers provide. Using this technique, future work could compare the effects of smoothing seed layer stacks such as Ge/Ag versus $\mathrm{Cu} / \mathrm{Ag}$ to find quantifiable differences in EME.

\subsubsection{NSDG quality}

The NSDG efficiency is an important quantity to the FSL, and optimising this could lead to much better imaging. The grating efficiency is maximised by reducing harmonics from the laser exposure so that only one grating pitch is exposed. This is compared to the current setup which results in increasing harmonic pitches at the microscale. This could be 
solved in future by using digital spatial filters [117].

Furthermore, BARC etching can be improved with an intermediary layer of silicon oxide $\left(\mathrm{SiO}_{2}\right)$ between the photoresist and BARC. This will allow much greater selective development of the photoresist, with the $\mathrm{SiO}_{2}$ and BARC remaining untouched. Then, the $\mathrm{SiO}_{2}$ can be etched using sulfur hexafluoride $\left(\mathrm{SF}_{6}\right)$ in RIE, and finally an $\mathrm{O}_{2}$ plasma to etch the BARC layer. This method is known as SiARC $[118,119]$ and would effectively allow up to $500 \mathrm{~nm}$ deep $200 \mathrm{~nm}$ pitch BARC gratings. The height of the gratings, along with the isotropic etch which the RIE provides, could produce gratings with very low LWR, greatly improving grating efficiency. It should be noted that the SiARC method was not explored within this thesis due to lack of access to required equipment, including an $\mathrm{SF}_{6}$-based RIE system.

\subsubsection{Far field superlens}

In the simplified design outline in Section 3.3 and again in Figure 6.3, the metal gratings must first be created on the substrate, then filled with PMMA. Planarisation of the PMMA is required as it is difficult to create an exact thickness of PMMA, but mainly spin coating a viscous liquid onto a non-uniform film causes issues of its own. Due to the gratings and surface tension, the PMMA will not easily fill the gaps between the gratings. To coerce the PMMA into the gaps, an overly thick layer of PMMA must be spun on. Planarisation can be performed in a low power $\mathrm{O}_{2}$ plasma, which effectively etches specific thickness of PMMA equal to the amount of time it is exposed to the plasma. Furthermore, the planarisation will also produce a very smooth PMMA surface.

From this point, using the methods outlined previously, it should be possible for a future researcher to create functional Ag gratings on a borosilicate substrate, fill and planarise PMMA, and finally top with an Ag film to produce an FSL. 


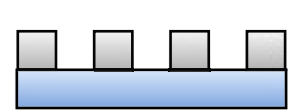

(a)

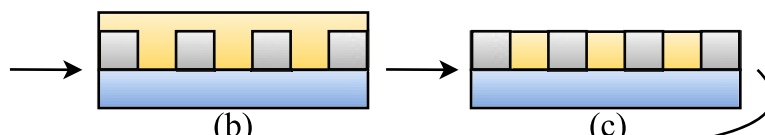

(b) (c)

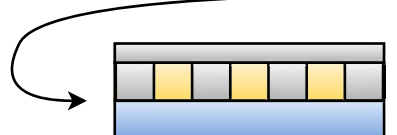

(d)

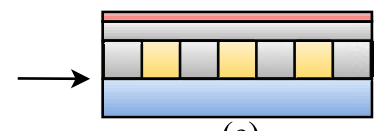

(e)

Figure 6.3: Simplified fabrication steps for the FSL: (a) create metal gratings on substrate, (b) add PMMA backfill, (c) planarise PMMA, (d) addition of Ag film, and (e) capping layer.

The fabrication steps outlined in this thesis lead to a considerable improvement in attainable devices with limited equipment. Furthermore, the advancements made to fabrication have also resulted in improved device performance. Therefore, this thesis paves the way to improved fabrication of sub-nanometer smooth films and sub-wavelength features for use in photonic devices, to further increase the performance of relevant devices, such as photonic sensors and solar cells, and optical microscopes. 


\section{Bibliography}

[1] J. S. Deaver, Optical assembly of far-field super-resolution imaging via optical gain. Western Carolina University, 2011.

[2] K. Catchpole and A. Polman, "Plasmonic solar cells," Optics Epress, vol. 16, no. 26, pp. 21793-21800, 2008.

[3] E. Ozbay, "Plasmonics: merging photonics and electronics at nanoscale dimensions," Science, vol. 311, no. 5758, pp. 189-193, 2006.

[4] J. B. Pendry, "Negative refraction makes a perfect lens," Physical Review Letters, vol. 85, no. 18, p. 3966, 2000.

[5] T. Stefaniuk, P. Wróbel, P. Trautman, and T. Szoplik, “Ultrasmooth metal nanolayers for plasmonic applications: surface roughness and specific resistivity," Applied Optics, vol. 53, no. 10, pp. B237-B241, 2014.

[6] N. Formica, D. S. Ghosh, A. Carrilero, T. L. Chen, R. E. Simpson, and V. Pruneri, "Ultrastable and atomically smooth ultrathin silver films grown on a copper seed layer," ACS Applied Materials \& Interfaces, vol. 5, no. 8, pp. 3048-3053, 2013.

[7] V. A. Podolskiy and E. E. Narimanov, "Near-sighted superlens," $O p$ tics Letters, vol. 30, no. 1, pp. 75-77, 2005.

[8] S. Durant, Z. Liu, J. M. Steele, and X. Zhang, "Theory of the transmission properties of an optical far-field superlens for imaging be- 
yond the diffraction limit," JOSA B, vol. 23, no. 11, pp. 2383-2392, 2006.

[9] J. Vigoureux and D. Courjon, "Detection of nonradiative fields in light of the heisenberg uncertainty principle and the rayleigh criterion," Applied Optics, vol. 31, no. 16, pp. 3170-3177, 1992.

[10] J. Kijowski, "On the time operator in quantum mechanics and the heisenberg uncertainty relation for energy and time," Reports on Mathematical Physics, vol. 6, no. 3, pp. 361-386, 1974.

[11] L. Rayleigh, "XXXI. investigations in optics, with special reference to the spectroscope," The London, Edinburgh, and Dublin Philosophical Magazine and Journal of Science, vol. 8, no. 49, pp. 261-274, 1879.

[12] K. K. Shung, Diagnostic ultrasound: Imaging and blood flow measurements. CRC press, 2005.

[13] M. Born and E. Wolf, Principles of optics: electromagnetic theory of propagation, interference and diffraction of light. Cambridge university press, 1999.

[14] V. N. Hulst, M. Moers, and B. Bölger, "Near-field optical microscopy in transmission and reflection modes in combination with force microscopy," Journal of Microscopy, vol. 171, no. 2, pp. 95-105, 1993.

[15] Z. Liu, S. Durant, H. Lee, Y. Pikus, N. Fang, Y. Xiong, C. Sun, and X. Zhang, "Far-field optical superlens," Nano Letters, vol. 7, no. 2, pp. 403-408, 2007.

[16] J. B. Pendry and D. R. Smith, "Reversing light with negative refraction," Physics Today, vol. 57, pp. 37-43, 2004.

[17] Aluminium, "Representation of an evanescent wave and a diffracted wave at an interface.." http://commons.wikimedia. org/wiki/File:Evanescent_wave.jpg [accessed 5 Jun. 2015]. 
[18] C. M. Krowne and Y. Zhang, Physics of Negative Refraction and Negative Index Materials. Springer, 2007.

[19] V. G. Veselago, "The electrodynamics of substances with simultaneously negative values of $\epsilon$ and $\mu$," Physics-Uspekhi, vol. 10, no. 4, pp. 509-514, 1968.

[20] A. Grigorenko, A. Geim, H. Gleeson, Y. Zhang, A. Firsov, I. Khrushchev, and J. Petrovic, "Nanofabricated media with negative permeability at visible frequencies," Nature, vol. 438, no. 7066, pp. 335-338, 2005.

[21] D. R. Smith, J. B. Pendry, and M. C. Wiltshire, "Metamaterials and negative refractive index," Science, vol. 305, no. 5685, pp. 788-792, 2004.

[22] D. Mills and E. Burstein, "Polaritons: the electromagnetic modes of media," Reports on Progress in Physics, vol. 37, no. 7, p. 817, 1974.

[23] K. Welford, "Surface plasmon-polaritons and their uses," Optical and Quantum Electronics, vol. 23, no. 1, pp. 1-27, 1991.

[24] E. Le Ru and P. Etchegoin, Principles of Surface-Enhanced Raman Spectroscopy: and related plasmonic effects. Elsevier, 2008.

[25] J. Pendry, L. Martin-Moreno, and F. Garcia-Vidal, "Mimicking surface plasmons with structured surfaces," Science, vol. 305, no. 5685, pp. 847-848, 2004.

[26] H. T. Baltar, E. M. Goldys, and K. Drozdowicz-Tomsia, Propagating surface plasmons and dispersion relations for nanoscale multilayer metallic-dielectric films. INTECH Open Access Publisher, 2012.

[27] A. D. Rakić, A. B. Djurišić, J. M. Elazar, and M. L. Majewski, “Optical properties of metallic films for vertical-cavity optoelectronic devices," Applied Optics, vol. 37, no. 22, pp. 5271-5283, 1998. 
[28] P. A. Van Der Merwe, “Surface plasmon resonance,” 2001.

[29] J. Homola, S. S. Yee, and G. Gauglitz, "Surface plasmon resonance sensors: review," Sensors and Actuators B: Chemical, vol. 54, no. 1, pp. 3-15, 1999.

[30] Y. Lu, W. Zhang, and M. Qiu, "Metamaterials, plasmonics, and THz frequency photonic components," Active and Passive Electronic Components, vol. 2007, 2008.

[31] H. Raether, Surface plasmons on smooth surfaces. Springer, 1988.

[32] E. Kretschmann, T. Ferrell, and J. Ashley, "Splitting of the dispersion relation of surface plasmons on a rough surface," Physical Review Letters, vol. 42, no. 19, p. 1312, 1979.

[33] E. Altewischer, M. Van Exter, and J. Woerdman, "Plasmon-assisted transmission of entangled photons," Nature, vol. 418, no. 6895, pp. 304-306, 2002.

[34] D. R. Smith, "How to build a superlens," Science, vol. 308, no. 5721, pp. 502-503, 2005.

[35] N. Fang, H. Lee, C. Sun, and X. Zhang, "Sub-diffraction-limited optical imaging with a silver superlens," Science, vol. 308, no. 5721, pp. 534-537, 2005.

[36] I. I. Smolyaninov, Y.-J. Hung, and C. C. Davis, "Magnifying superlens in the visible frequency range," Science, vol. 315, no. 5819, pp. 1699-1701, 2007.

[37] Z. Liu, "Far-field superlens for optical imaging beyond diffraction limit and other nano-plasmonic devices," 2006.

[38] H. W. Ott and H. W. Ott, Noise reduction techniques in electronic systems, vol. 442. Wiley New York, 1988. 
[39] Z. Liu, S. Durant, H. Lee, Y. Pikus, Y. Xiong, C. Sun, and X. Zhang, "Experimental studies of far-field superlens for sub-diffractional optical imaging," Optics Express, vol. 15, no. 11, pp. 6947-6954, 2007.

[40] Y. Xiong, Z. Liu, S. Durant, H. Lee, C. Sun, and X. Zhang, “Tuning the far-field superlens: from uv to visible," Optics Express, vol. 15, no. 12, pp. 7095-7102, 2007.

[41] S. A. Maier, M. L. Brongersma, P. G. Kik, and H. A. Atwater, "Observation of near-field coupling in metal nanoparticle chains using far-field polarization spectroscopy," Physical Review B, vol. 65, no. 19, p. 193408, 2002.

[42] Z. Liu, H. Lee, Y. Xiong, C. Sun, and X. Zhang, "Far-field optical hyperlens magnifying sub-diffraction-limited objects," Science, vol. 315, no. 5819, pp. 1686-1686, 2007.

[43] R. Verma and H. Lee, "Far-field superlensing," Feb. 4 2010. US Patent App. 12/658,342.

[44] Z. Jacob, L. V. Alekseyev, and E. Narimanov, “Optical hyperlens: farfield imaging beyond the diffraction limit," Optics Express, vol. 14, no. 18, pp. 8247-8256, 2006.

[45] R. Swanepoel, "Determination of surface roughness and optical constants of inhomogeneous amorphous silicon films," Journal of Physics E: Scientific Instruments, vol. 17, no. 10, p. 896, 1984.

[46] S. A. Maier, Plasmonics: fundamentals and applications. Springer Science \& Business Media, 2007.

[47] "Surface roughness." http://www.olympus-ims.com/en/ knowledge/metrology/roughness/. (Visited on 27/07/2015). 
[48] H. Namatsu, Y. Takahashi, K. Yamazaki, T. Yamaguchi, M. Nagase, and K. Kurihara, "Three-dimensional siloxane resist for the formation of nanopatterns with minimum linewidth fluctuations," Journal of Vacuum Science E Technology B, vol. 16, no. 1, pp. 69-76, 1998.

[49] N. Fang, Z. Liu, T.-J. Yen, and X. Zhang, "Regenerating evanescent waves from a silver superlens," Optics Express, vol. 11, no. 7, pp. 682687, 2003.

[50] X. Rao and C. Ong, "Subwavelength imaging by a left-handed material superlens," Physical Review E, vol. 68, no. 6, p. 067601, 2003.

[51] T. Taubner, D. Korobkin, Y. Urzhumov, G. Shvets, and R. Hillenbrand, "Near-field microscopy through a SiC superlens," Science, vol. 313, no. 5793, pp. 1595-1595, 2006.

[52] C. Luo, S. G. Johnson, J. Joannopoulos, and J. Pendry, "Subwavelength imaging in photonic crystals," Physical Review B, vol. 68, no. 4, p. $045115,2003$.

[53] R. Moussa, S. Foteinopoulou, L. Zhang, G. Tuttle, K. Guven, E. Ozbay, and C. Soukoulis, "Negative refraction and superlens behavior in a two-dimensional photonic crystal," Physical Review B, vol. 71, no. 8, p. 085106, 2005.

[54] Y. Xiong, Z. Liu, C. Sun, and X. Zhang, "Two-dimensional imaging by far-field superlens at visible wavelengths," Nano Letters, vol. 7, no. 11, pp. 3360-3365, 2007.

[55] E. Kröger and E. Kretschmann, "Scattering of light by slightly rough surfaces or thin films including plasma resonance emission," Zeitschrift für Physik, vol. 237, no. 1, pp. 1-15, 1970.

[56] N. Fang, Z. Liu, T.-J. Yen, and X. Zhang, “Experimental study of transmission enhancement of evanescent waves through silver films 
assisted by surface plasmon excitation," Applied Physics A, vol. 80, no. 6, pp. 1315-1325, 2005.

[57] W. Chen, K. Chen, M. D. Thoreson, A. Kildishev, and V. M. Shalaev, "Ultrathin, ultrasmooth, and low-loss silver films via wetting and annealing," Applied Physics Letters, vol. 97, no. 21, pp. 2-11,107, 2010.

[58] J. Tominaga, "The application of silver oxide thin films to plasmon photonic devices," Journal of Physics: Condensed Matter, vol. 15, no. 25, p. R1101, 2003.

[59] S. Kawata, Y. Inouye, and P. Verma, "Plasmonics for near-field nanoimaging and superlensing," Nature Photonics, vol. 3, no. 7, pp. 388394, 2009.

[60] H. Lee, Z. Liu, Y. Xiong, C. Sun, and X. Zhang, "Design, fabrication and characterization of a far-field superlens," Solid State Communications, vol. 146, no. 5, pp. 202-207, 2008.

[61] M. J. Word, I. Adesida, and P. R. Berger, “Nanometer-period gratings in hydrogen silsesquioxane fabricated by electron beam lithography," Journal of Vacuum Science \& Technology B, vol. 21, no. 6, pp. L12L15, 2003.

[62] H. Solak, C. David, J. Gobrecht, L. Wang, and F. Cerrina, "Multiplebeam interference lithography with electron beam written gratings," Journal of Vacuum Science \& Technology B, vol. 20, no. 6, pp. 2844-2848, 2002.

[63] H. Lüth, Solid surfaces, interfaces and thin films, vol. 4. Springer, 2001.

[64] V. M. Shalaev, Optical properties of nanostructured random media, vol. 82. Springer Science \& Business Media, 2002.

[65] V. Logeeswaran, N. P. Kobayashi, M. S. Islam, W. Wu, P. Chaturvedi, N. X. Fang, S. Y. Wang, and R. S. Williams, “Ultrasmooth silver thin 
films deposited with a germanium nucleation layer," Nano Letters, vol. 9, no. 1, pp. 178-182, 2008.

[66] P. Nyga, V. Drachev, M. D. Thoreson, and V. Shalaev, "Mid-IR plasmonics and photomodification with Ag films," Applied Physics B, vol. 93, no. 1, pp. 59-68, 2008.

[67] R. Jaccodine, "Surface energy of germanium and silicon," Journal of The Electrochemical Society, vol. 110, no. 6, pp. 524-527, 1963.

[68] V. Goleus, A. Y. Belyi, É. Sardak, and Y. I. Belyi, "Calculation of the surface tension of molten borosilicate glasses," Glass and Ceramics, vol. 53, no. 8, pp. 226-228, 1996.

[69] J. N. Israelachvili, Intermolecular and surface forces: revised third edition. Academic press, 2011.

[70] W. Chen, M. D. Thoreson, S. Ishii, A. V. Kildishev, and V. M. Shalaev, "Ultra-thin ultra-smooth and low-loss silver films on a germanium wetting layer," Optics Express, vol. 18, no. 5, pp. 5124-5134, 2010.

[71] X. Zhang and Z. Liu, "Superlenses to overcome the diffraction limit," Nature Materials, vol. 7, no. 6, pp. 435-441, 2008.

[72] J. R. Heath, J. Shiang, and A. Alivisatos, "Germanium quantum dots: Optical properties and synthesis," The Journal of Chemical physics, vol. 101, no. 2, pp. 1607-1615, 1994.

[73] Y. Mo, I. Mörke, and P. Wachter, "Surface enhanced raman scattering of pyridine on silver surfaces of different roughness," Surface Science Letters, vol. 133, no. 1, pp. L452-L458, 1983.

[74] J. Schroeder and J. H. Rosolowski, "Light scattering in polycrystalline materials," in 25th Annual Technical Symposium, pp. 156-168, International Society for Optics and Photonics, 1982. 
[75] L. Vitos, A. Ruban, H. L. Skriver, and J. Kollar, “The surface energy of metals," Surface Science, vol. 411, no. 1, pp. 186-202, 1998.

[76] Y. Chiu, U. Rambabu, M.-H. Hsu, H.-P. D. Shieh, C.-Y. Chen, H.-H. Lin, et al., "Fabrication and nonlinear optical properties of nanoparticle silver oxide films," Journal of Applied Physics, vol. 94, no. 3, pp. 1996-2001, 2003.

[77] J. P. Allen, D. O. Scanlon, and G. W. Watson, "Electronic structures of silver oxides," Physical Review B, vol. 84, no. 11, p. 115141, 2011.

[78] F. Bock, T. Christensen, S. Rivers, L. Doucette, and R. Lad, "Growth and structure of silver and silver oxide thin films on sapphire," Thin Solid Films, vol. 468, no. 1, pp. 57-64, 2004.

[79] K. Thyagarajan, J. Butet, and O. J. Martin, "Augmenting second harmonic generation using fano resonances in plasmonic systems," Nano Letters, vol. 13, no. 4, pp. 1847-1851, 2013.

[80] D. Flötotto, Z. Wang, L. P. Jeurgens, and E. Mittemeijer, "Intrinsic stress evolution during amorphous oxide film growth on $\mathrm{Al}$ surfaces," Applied Physics Letters, vol. 104, no. 9, p. 091901, 2014.

[81] M. Puchert, P. Timbrell, and R. Lamb, "Postdeposition annealing of radio frequency magnetron sputtered $\mathrm{ZnO}$ films," Journal of Vacuum Science E Technology A, vol. 14, no. 4, pp. 2220-2230, 1996.

[82] F. dHeurle, "Aluminum films deposited by RF sputtering," Metallurgical and Materials Transactions B, vol. 1, no. 3, pp. 725-732, 1970.

[83] AZ Electronic Materials, "AZ1500 photoresist data package." http://photoresist.com/wp-content/uploads/2010/ 11/az_1500_photoresist.pdf [24 Feb. 2015].

[84] R. Voelkel, U. Vogler, A. Bich, P. Pernet, K. J. Weible, M. Hornung, R. Zoberbier, E. Cullmann, L. Stuerzebecher, T. Harzendorf, et al., 
"Advanced mask aligner lithography: new illumination system," Optics Epress, vol. 18, no. 20, pp. 20968-20978, 2010.

[85] A. Bramati, U. Vogler, B. Meliorisz, K. Motzek, M. Hornung, and R. Voelkel, "Simulation tools for advanced mask aligner lithography," in SPIE Optical Systems Design, pp. 81670U-81670U, International Society for Optics and Photonics, 2011.

[86] C. P. Moore, "Optical superlenses: Quality and fidelity in silverdielectric near-field imaging systems," University of Canterbury. Electrical and Computer Engineering Department, p. 127, 2012.

[87] Thorlabs, "S120vc - standard photodiode power sensor, si, 200 - 1100 nm, 50 mw." https: //www.thorlabs.com/thorcat/18300/ S120VC-AutoCADPDF . pdf [24 Nov. 2015].

[88] M. J. Madou, Manufacturing techniques for microfabrication and nanotechnology, vol. 2. CRC Press, 2011.

[89] L. J. Guo, "Nanoimprint lithography: methods and material requirements," ADVANCED MATERIALS-DEERFIELD BEACH THEN WEINHEIM-, vol. 19, no. 4, p. 495, 2007.

[90] Y. C. Bae, K. Douki, T. Yu, J. Dai, D. Schmaljohann, H. Koerner, C. K. Ober, and W. Conley, "Tailoring transparency of imageable fluoropolymers at $157 \mathrm{~nm}$ by incorporation of hexafluoroisopropyl alcohol to photoresist backbones," Chemistry of Materials, vol. 14, no. 3, pp. 1306-1313, 2002.

[91] H. Solak, D. He, W. Li, and F. Cerrina, "Nanolithography using extreme ultraviolet lithography interferometry: $19 \mathrm{~nm}$ lines and spaces," Journal of Vacuum Science E Technology B, vol. 17, no. 6, pp. 3052-3057, 1999. 
[92] M. N. Polyanskiy, "Refractive index of $\left(\mathrm{c}_{5} \mathrm{O}_{2} \mathrm{~h}_{8}\right)_{\mathrm{n}}$ (poly)methyl methacrylate (pmma) - szczurowski." http: //refractiveindex. info/ ?shelf=organic\&book= poly (methyl_methacrylate) \&page=Szczurowski. (Visited on $02 / 18 / 2016)$.

[93] P. Gadenne, D. Gagnot, and M. Masson, "Surface enhanced resonant raman scattering induced by silver thin films close to the percolation threshold," Physica A: Statistical Mechanics and its Applications, vol. 241, no. 1, pp. 161-165, 1997.

[94] H. Lee, Y. Xiong, N. Fang, W. Srituravanich, S. Durant, M. Ambati, C. Sun, and X. Zhang, "Realization of optical superlens imaging below the diffraction limit," New Journal of Physics, vol. 7, no. 1, p. 255, 2005.

[95] M. Ackerman, F. E. Stafford, and J. Drowart, "Mass spectrometric determination of the dissociation energies of the molecules $\mathrm{AgAu}$, $\mathrm{AgCu}$, and $\mathrm{AuCu}$," The Journal of Chemical physics, vol. 33, no. 6, pp. 1784-1789, 1960.

[96] H. Lee, D. Kwon, H. Park, H. W. Kim, C. Lee, and J. Lee, "Rapid thermal annealing treatment of electroplated Cu films," JOURNALKOREAN PHYSICAL SOCIETY, vol. 43, no. 2, pp. 841-846, 2003.

[97] Y. Tsuda, H. Omoto, K. Tanaka, and H. Ohsaki, "The underlayer effects on the electrical resistivity of Ag thin film," Thin Solid Films, vol. 502, no. 1, pp. 223-227, 2006.

[98] W. Tang, K. Xu, P. Wang, and X. Li, "Surface roughness and resistivity of au film on Si-(111) substrate," Microelectronic engineering, vol. 66, no. 1, pp. 445-450, 2003. 
[99] Y. Ke, F. Zahid, V. Timoshevskii, K. Xia, D. Gall, and H. Guo, "Resistivity of thin $\mathrm{Cu}$ films with surface roughness," Physical Review B, vol. 79, no. 15, p. 155406, 2009.

[100] R. B. Stephens and G. D. Cody, "Optical reflectance and transmission of a textured surface," Thin Solid Films, vol. 45, no. 1, pp. 19-29, 1977.

[101] H. Kim, T. Alford, and D. Allee, "Thickness dependence on the thermal stability of silver thin films," Applied Physics Letters, vol. 81, no. 22, pp. 4287-4289, 2002.

[102] R. Dannenberg, E. Stach, J. R. Groza, and B. J. Dresser, “TEM annealing study of normal grain growth in silver thin films," Thin Solid Films, vol. 379, no. 1, pp. 133-138, 2000.

[103] I. Malitson and M. Dodge, "Refractive-index and birefringence of synthetic sapphire," in Journal Of The Optical Society Of America, vol. 62, pp. 1405-1405, AMER INST PHYSICS, 1972.

[104] R. Scorpio, Fundamentals of acids, bases, buffers and their application to biochemical systems. Kendall/Hunt Publishing Company, 2000.

[105] Microchemicals, "General properties of AZ/TI photoresists datasheet." http://www.microchemicals.com/technical_ information/photoresist_properties.pdf. (Visited on 02/16/2016).

[106] A. Jouyban, M. A. A. Fakhree, and A. Shayanfar, "Review of pharmaceutical applications of n-methyl-2-pyrrolidone," Journal of Pharmacy \& Pharmaceutical Sciences, vol. 13, no. 4, pp. 524-535, 2010.

[107] Microchemicals, "Nano remover PG datasheet." http://www . microchem. com/pdf/removerpg.pdf. (Visited on 02/16/2016).

[108] S. Garanin, F. Starikov, and Y. I. Malakhov, Adaptive Optics and Optical Vortices. INTECH Open Access Publisher, 2012. 
[109] M. Araghchini, Y. Yeng, N. Jovanovic, P. Bermel, L. Kolodziejski, M. Soljacic, I. Celanovic, and J. Joannopoulos, "Fabrication of twodimensional tungsten photonic crystals for high-temperature applications," Journal of Vacuum Science \& Technology B, vol. 29, no. 6, p. 061402, 2011.

[110] G. S. Oehrlein, "Reactive-ion etching," Physics Today, vol. 39, no. 10, pp. 26-33, 2008.

[111] F. Villa, L. Regalado, F. Ramos-Mendieta, J. Gaspar-Armenta, and T. Lopez-Ríos, "Photonic crystal sensor based on surface waves for thin-film characterization," Optics Letters, vol. 27, no. 8, pp. 646-648, 2002.

[112] U. Willer and W. Schade, "Photonic sensor devices for explosive detection," Analytical and bioanalytical chemistry, vol. 395, no. 2, pp. 275282, 2009.

[113] P. Bermel, C. Luo, L. Zeng, L. C. Kimerling, and J. D. Joannopoulos, "Improving thin-film crystalline silicon solar cell efficiencies with photonic crystals," Optics Express, vol. 15, no. 25, pp. 16986-17000, 2007.

[114] D. Zhou and R. Biswas, "Photonic crystal enhanced light-trapping in thin film solar cells," Journal of Applied Physics, vol. 103, no. 9, p. $093102,2008$.

[115] S. H. Park, A. Roy, S. Beaupre, S. Cho, N. Coates, J. S. Moon, D. Moses, M. Leclerc, K. Lee, and A. J. Heeger, "Bulk heterojunction solar cells with internal quantum efficiency approaching $100 \%$," nature photonics, vol. 3, no. 5, pp. 297-302, 2009.

[116] A. Hatta, Y. Chiba, and W. Suëtaka, "Infrared absorption study of adsorbed species at metal/water interface by use of the 
kretschmann configuration," Surface Science, vol. 158, no. 1, pp. 616623, 1985.

[117] F. Dubois, M.-L. N. Requena, C. Minetti, O. Monnom, and E. Istasse, "Partial spatial coherence effects in digital holographic microscopy with a laser source," Applied Optics, vol. 43, no. 5, pp. 1131-1139, 2004.

[118] M. G. Wood, L. Chen, J. R. Burr, and R. M. Reano, “Optimization of electron beam patterned hydrogen silsesquioxane mask edge roughness for low-loss silicon waveguides," Journal of Nanophotonics, vol. 8, no. 1, pp. 083098-083098, 2014.

[119] T. Savas, M. Schattenburg, J. Carter, and H. I. Smith, "Large-area achromatic interferometric lithography for $100 \mathrm{~nm}$ period gratings and grids," Journal of Vacuum Science E Technology B, vol. 14, no. 6, pp. 4167-4170, 1996. 